\title{
Chaotic Escape From an Open Vase-Shaped Cavity. II. Topological Theory
}

Jaison Allen Novick

William \& Mary

John B. Delos

William \& Mary, jbdelos@wm.edu

Follow this and additional works at: https://scholarworks.wm.edu/aspubs

Part of the Physics Commons

\section{Recommended Citation}

Novick, Jaison Allen and Delos, John B., Chaotic Escape From an Open Vase-Shaped Cavity. II. Topological Theory (2012). Physical Review E, 85(1).

https://doi.org/10.1103/PhysRevE.85.016206

This Article is brought to you for free and open access by the Arts and Sciences at W\&M ScholarWorks. It has been accepted for inclusion in Arts \& Sciences Articles by an authorized administrator of W\&M ScholarWorks. For more information, please contact scholarworks@wm.edu. 


\title{
Chaotic escape from an open vase-shaped cavity. II. Topological theory
}

\author{
Jaison Novick and John B. Delos* \\ Department of Physics, College of William and Mary, Williamsburg, Virginia 23187-8795, USA
}

(Received 7 November 2010; published 18 January 2012)

\begin{abstract}
We present part II of a study of chaotic escape from an open two-dimensional vase-shaped cavity. A surface of section reveals that the chaotic dynamics is controlled by a homoclinic tangle, the union of stable and unstable manifolds attached to a hyperbolic fixed point. Furthermore, the surface of section rectifies escape-time graphs into sequences of escape segments; each sequence is called an epistrophe. Some of the escape segments (and therefore some of the epistrophes) are forced by the topology of the dynamics of the homoclinic tangle. These topologically forced structures can be predicted using the method called homotopic lobe dynamics (HLD). HLD takes a finite length of the unstable manifold and a judiciously altered topology and returns a set of symbolic dynamical equations that encode the folding and stretching of the unstable manifold. We present three applications of this method to three different lengths of the unstable manifold. Using each set of dynamical equations, we compute minimal sets of escape segments associated with the unstable manifold, and minimal sets associated with a burst of trajectories emanating from a point on the vase's boundary. The topological theory predicts most of the early escape segments that are found in numerical computations.
\end{abstract}

DOI: 10.1103/PhysRevE.85.016206

PACS number(s): 05.45.Df, 02.40.Re, 05.60.Cd, 42.15.Dp

\section{INTRODUCTION}

This is the second in a pair of papers studying chaotic escape of rays from a vase-shaped cavity [1]. The importance of chaotic transport in a variety of fields was discussed in paper I. We explained that an open two-dimensional cavity having the shape of a vase provides a simple model system having all the complexity that is characteristic of chaotic transport. There we also showed, calculated, and measured time spectra of escaping orbits for a vase having a particular shape. We showed that a graph of escape time vs initial direction of motion from a source point consists of infinitely long sequences of "icicles," and every icicle has an infinite sequence of icicles converging upon its boundary. Therefore the graph has fractal structure-within-structure at all levels of resolution.

Recently a number of workers have developed topological methods to understand and predict the fractal structure of such graphs [2-35]. Often the method involves computation of a surface of section up to some number of iterates, and then predicting properties that are topologically forced for higher iterates. A formulation that is especially well adapted for studying electrons escaping from atoms in electric and magnetic fields, and for studying rays escaping from a vase, was developed in Refs. [23-32]. More precisely, three closely related formulations were given in those references, with the more recent formulations being more powerful but more complex. In previous work on the vase, we used the method given in [24]. Here we use the "bridges" method presented in Ref. [27] to analyze the fractal structure that appears in escape from a vase.

The paper is organized as follows. Section II gives a brief review of homoclinic tangles and chaotic transport. Section III describes homotopic lobe dynamics, the topological method we will apply. Section IV presents applications of homotopic lobe dynamics. Finally, Sec. V gives topological entropies for our applications and a discussion of our results.

\footnotetext{
*jbdelo@wm.edu
}

\section{HOMOCLINIC TANGLES}

We begin our discussion by describing how the homoclinic tangle controls chaotic transport. We present a simple example that highlights how a homoclinic tangle stretches and folds sets of points and introduces basic definitions.

\section{A. Preliminaries}

Here we describe the essential aspects of homoclinic tangles [23,24,33]. As described in the accompanying paper [1], the surface of section for the vase has an unstable fixed point with stable manifold $S$ and unstable manifold $\boldsymbol{u}$. A closed segment of either manifold with end points $\boldsymbol{x}_{\mathbf{1}}, \boldsymbol{x}_{\mathbf{2}}$ is denoted by $S\left[x_{1}, x_{2}\right]$ or $\mathcal{u}\left[x_{1}, x_{2}\right]$, where the ordering of the points denotes the natural orientation of the manifold. For example, the segments of $\boldsymbol{U}$ and $\boldsymbol{S}$ connecting the points $\boldsymbol{q}_{\boldsymbol{x}}$ and $\boldsymbol{P}_{\mathbf{0}}$ in Fig. 1(a) are denoted separately as $\boldsymbol{U}\left[\boldsymbol{q}_{\boldsymbol{x}}, \boldsymbol{P}_{\mathbf{0}}\right]$ and $\boldsymbol{S}\left[\boldsymbol{P}_{\mathbf{0}}, \boldsymbol{q}_{\boldsymbol{x}}\right]$. If we are considering both the stable and unstable segments connecting $x_{1}$ to $x_{2}$, then for convenience we let $\boldsymbol{u} / \mathcal{S}\left[\boldsymbol{x}_{1}, \boldsymbol{x}_{2}\right]$ denote the phrase " $\boldsymbol{u}\left[\boldsymbol{x}_{1}, \boldsymbol{x}_{2}\right]$ and $S\left[\boldsymbol{x}_{1}, \boldsymbol{x}_{2}\right]$ " and ignore the ordering of the points. We call the closed eye-shaped region in Fig. 1(a) the "complex," denoted by $\Gamma$. The boundary of $\Gamma$ is called $\boldsymbol{\Gamma}_{\boldsymbol{s} / \boldsymbol{u}}=\boldsymbol{u} / \boldsymbol{S}\left[\boldsymbol{q}_{\boldsymbol{x}}, \boldsymbol{P}_{\mathbf{0}}\right]$. We are concerned with how points exit the complex. Points that escape the complex never reenter it.

The points $\boldsymbol{P}_{\mathbf{0}}$ and $\boldsymbol{Q}_{\mathbf{0}}$ are homoclinic points, which generate homoclinic orbits, $\boldsymbol{P}_{\boldsymbol{n}}=\boldsymbol{M}^{n}\left(\boldsymbol{P}_{\mathbf{0}}\right)$ and $\boldsymbol{Q}_{\boldsymbol{n}}=\boldsymbol{M}^{n}\left(\boldsymbol{Q}_{\mathbf{0}}\right)$ where $n$ is any integer. We call the region bounded by $\boldsymbol{S}\left[\boldsymbol{P}_{\boldsymbol{n}}, \boldsymbol{Q}_{\boldsymbol{n}}\right]$ and $\boldsymbol{U}\left[\boldsymbol{P}_{\boldsymbol{n}}, \boldsymbol{Q}_{\boldsymbol{n}}\right]$ the $n$th "escape lobe" $\mathrm{E}_{n}$. We call the region bounded by $\boldsymbol{S}\left[\boldsymbol{Q}_{\boldsymbol{n}-1}, \boldsymbol{P}_{\boldsymbol{n}}\right]$ and $\mathcal{U}\left[\boldsymbol{Q}_{\boldsymbol{n}-1}, \boldsymbol{P}_{\boldsymbol{n}}\right]$ the $n$th "capture lobe" $\mathrm{C}_{n}$. We denote the unstable boundary of the $n$th capture lobe as $\boldsymbol{C}_{n}$. The dynamical mapping induces the relationships $\mathrm{E}_{n+1}=\boldsymbol{M}\left(\mathrm{E}_{n}\right)$ and $\mathrm{C}_{n+1}=\mathcal{M}\left(\mathrm{C}_{n}\right)$ where $n$ is any integer. The lobes $\mathrm{E}_{-1}, \mathrm{E}_{0}, \mathrm{C}_{0}$, and $\mathrm{C}_{1}$ together form what is known as a "turnstile." Points map into the complex by entering $\mathrm{C}_{0}$ and then mapping to $\mathrm{C}_{1}$; points exit the complex by entering $E_{-1}$ and then mapping to $E_{0}$. In Fig. 1(a), we see that $\mathrm{C}_{4}$ intersects the lobe $\mathrm{E}_{0}$. This means that once a point 

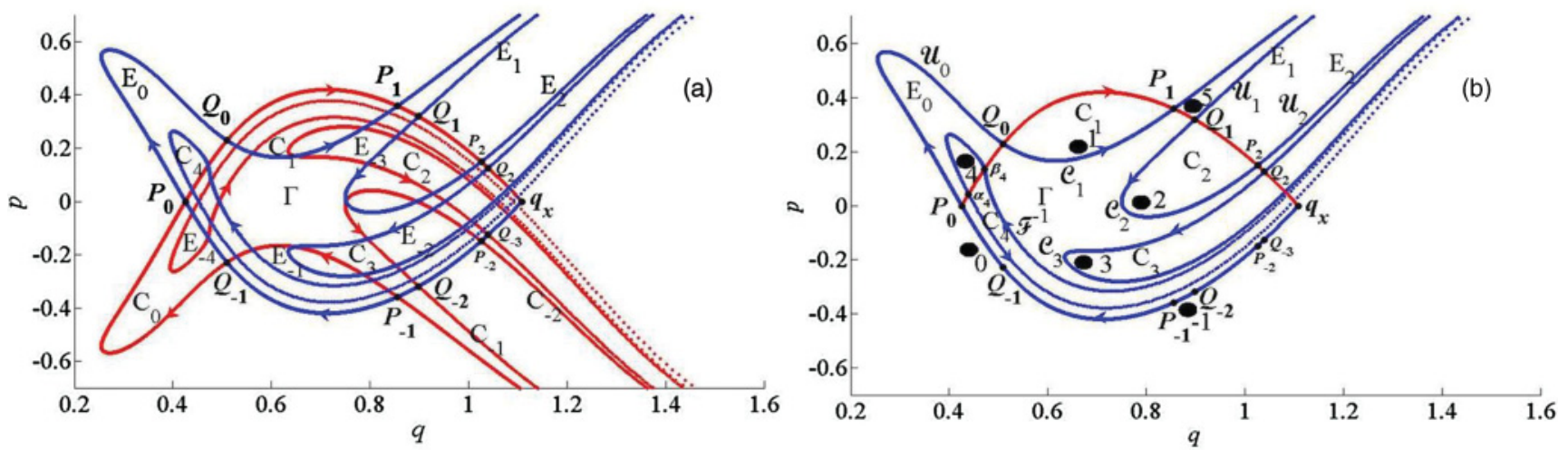

FIG. 1. (Color online) The homoclinic tangle underlying the dynamics in the vase. The stable and unstable manifolds are denoted $S$ and $\boldsymbol{U}$ [plotted in red and blue online (medium and dark gray), respectively]. The points $\boldsymbol{Q}_{n}=\boldsymbol{M}^{n}\left(\boldsymbol{Q}_{0}\right)$ and $\boldsymbol{P}_{\boldsymbol{n}}=\boldsymbol{M}^{\boldsymbol{n}}\left(\boldsymbol{P}_{\mathbf{0}}\right)$, where $n$ is any integer, are homoclinic points. The regions $\mathrm{E}_{n}$ and $\mathrm{C}_{n}$ are called escape and capture lobes, respectively. The complex, $\Gamma$, is the closed region bounded by the segments of $S$ and $\boldsymbol{U}$ connecting $\boldsymbol{q}_{\boldsymbol{x}}$ to $\boldsymbol{P}_{\mathbf{0}}, \boldsymbol{U} / \boldsymbol{S}\left[\boldsymbol{q}_{\boldsymbol{x}}, \boldsymbol{P}_{\mathbf{0}}\right]$ (a) Both manifolds, with several escape lobes and capture lobes labeled, are shown. (b) A selected length of the unstable manifold used in the topological analysis. The numbered disks represent holes, or regions through which curves cannot continuously move. The curves $\boldsymbol{C}_{1}, \boldsymbol{C}_{2}$, and $\boldsymbol{C}_{3}$ are portions of the unstable manifold which are boundaries of the lobes $\mathrm{C}_{1}$, $\mathrm{C}_{2}$, and $\mathrm{C}_{3}$, with $\boldsymbol{C}_{\boldsymbol{i}}=\boldsymbol{u}\left[\boldsymbol{Q}_{\boldsymbol{i}-1}, \boldsymbol{P}_{\boldsymbol{i}}\right]$. The unstable boundaries of the lobes $\mathrm{E}_{0}, \mathrm{E}_{1}$, and $\mathrm{E}_{2}$ are called $\boldsymbol{u}_{0}, \boldsymbol{u}_{1}$, and $\boldsymbol{u}_{2}$, with $\left.\boldsymbol{u}_{\boldsymbol{i}}=\boldsymbol{u}_{\boldsymbol{P}} \boldsymbol{P}_{\boldsymbol{i}}, \boldsymbol{Q}_{\boldsymbol{i}}\right]$.

has entered $\Gamma$, it must iterate at least three times to escape the complex. This integer is called the "minimum time delay $D . "$

\section{B. Homoclinic tangles and chaotic transport}

Figure 2(a) shows a qualitative tangle with minimum delay time of 1 . This means that points enter the complex by mapping from $\mathrm{C}_{0}$ to $\mathrm{C}_{1}$ and then can escape the complex by mapping into $\mathrm{E}_{0}$ after at least one iterate. Let us consider how $\mathrm{C}_{3}$ winds through the plane, which is shown in Fig. 2(b). The intersection of $\mathrm{C}_{2}$ and $\mathrm{E}_{0}$ maps to the intersection of $\mathrm{C}_{3}$ and $\mathrm{E}_{1}$. Neither manifold can cross itself; therefore, $\mathrm{C}_{3}$ is forced to wind under
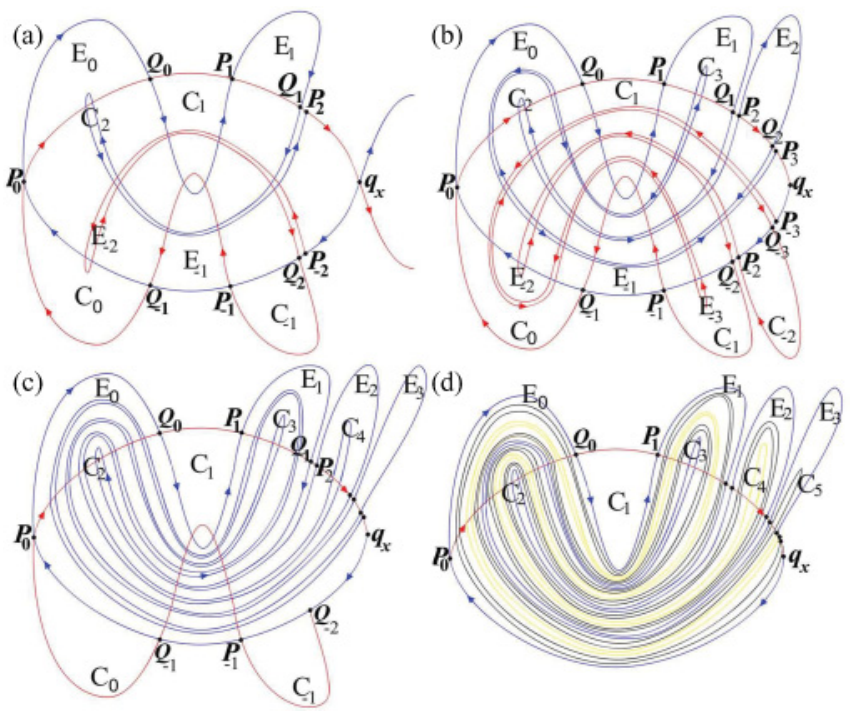

FIG. 2. (Color online) A qualitative homoclinic tangle with minimum delay time of 1 . This figure shows the qualitative dynamical evolution of the lobes $\mathrm{C}_{1}$ and $\mathrm{E}_{-1}$. We assume that the manifolds possess mirror symmetry about a horizontal axis connecting the points $\boldsymbol{P}_{\mathbf{0}}$ and $\boldsymbol{q}_{\boldsymbol{x}}$. (a) $\mathrm{C}_{1}, \mathrm{C}_{2}, \mathrm{E}_{-1}$, and $\mathrm{E}_{-2}$. (b) The same as (a) except $\mathrm{C}_{3}$ and $\mathrm{E}_{-3}$ have been added. (c) $\mathrm{E}_{-2}, \mathrm{E}_{-3}$, and $\mathrm{E}_{-4}$ have been removed but $\mathrm{C}_{4}$ has been added. (d) $\mathrm{C}_{1}, \mathrm{C}_{2}, \mathrm{C}_{3}$ [blue (gray)], $\mathrm{C}_{4}$ [yellow (light gray)], and $\mathrm{C}_{5}$ (black) are shown.
$\mathrm{C}_{2}$ and then over the tip of $\mathrm{C}_{2}$ as it passes through $\mathrm{E}_{0}$; it then winds under $C_{1}$ in order to intersect $E_{1}$. Now consider the image of $\mathrm{C}_{3}$, which is $\mathrm{C}_{4}$. The shape of $\mathrm{C}_{4}$ is determined by three facts: (1) $\mathrm{C}_{4}$ must intersect $\mathrm{E}_{2}$ (the intersection of $\mathrm{C}_{3}$ and $\mathrm{E}_{1}$ maps to the intersection of $\mathrm{C}_{4}$ and $\mathrm{E}_{2}$ ). (2) The points $\boldsymbol{Q}_{2}$ and $\boldsymbol{P}_{\mathbf{3}}$ map into $\boldsymbol{Q}_{\mathbf{3}}$ and $\boldsymbol{P}_{\mathbf{4}}$ which lie to closer to $\boldsymbol{q}_{\boldsymbol{x}}$ [they are shown but not labeled in Fig. 2(c)]. (3) $\boldsymbol{U}$ cannot cross over itself. Therefore, $\mathrm{C}_{4}$ is forced to wind under $\mathrm{C}_{3}$ until it enters $\mathrm{E}_{0}$, then over $\mathrm{C}_{3}$ as it passes through $\mathrm{E}_{0}$; then it must wind between $C_{3}$ and $C_{1}$ and over the tip of $C_{3}$ as it passes through $E_{1}$. In order for $C_{4}$ to intersect $E_{2}$, it must go back through $\mathrm{E}_{0}$ again, this time below $\mathrm{C}_{3}$ but above the tip of $\mathrm{C}_{2}$ in a counterclockwise manner. Finally, $\mathrm{C}_{4}$ winds under $\mathrm{C}_{2}$ to intersect $E_{2}$. (The evolution of the escape lobes is similar as we assume mirror symmetry of the manifolds.) We leave it to the reader to verify, using the same arguments, that $\mathrm{C}_{5}$, sketched in Fig. 2(d), is correctly drawn. The four pictures in Fig. 2 show that the mapping stretches and folds the capture lobes, leading to forced intersections with $\mathrm{E}_{0}$.

Let us now consider the effect of the mapping on a curve of points (Fig. 3). We consider the set $\mathcal{L}_{0}=$ $\left\{\left(q_{0}, p_{0}\right): q_{0}=0.5607\right.$ and $\left.p_{0} \in(-1,1)\right\}$. This vertical line segment represents a family of trajectories launched from a point on the vase boundary. The first, second, and third iterates of $\boldsymbol{L}_{0}$ are also plotted and they enclose $\boldsymbol{\mathcal { C }}_{1}$ (first iterate, green online), $\boldsymbol{C}_{2}$ (second iterate, yellow online), and $\boldsymbol{e}_{3}$ (third iterate, brown online), respectively. Figure 3 shows that as the burst evolves, the successive iterates of $\mathcal{L}_{0}$ inside the complex resemble the unstable boundaries of $\mathrm{C}_{1}, \mathrm{C}_{2}$, and $\mathrm{C}_{3}$. Figure 4 shows the fourth through seventh images of the burst. As in Fig. 3, these curves are similar to the corresponding iterates of $\boldsymbol{C}_{n}$. Specifically, we see that $\mathcal{L}_{4}$ passes once through $\mathrm{E}_{0}$, so one segment of the curve escapes from the complex on the fourth iterate, but $\mathcal{L}_{5}$ through $\mathcal{L}_{7}$ each pass through $\mathrm{E}_{0}$ twice so at each of these iterates, two segments of the curve escape from the complex. In the next section, we will exploit the similarities between iterates of $\mathcal{L}_{0}$ and $\boldsymbol{C}_{0}$ to predict how curves pass through $\mathrm{E}_{0}$. 


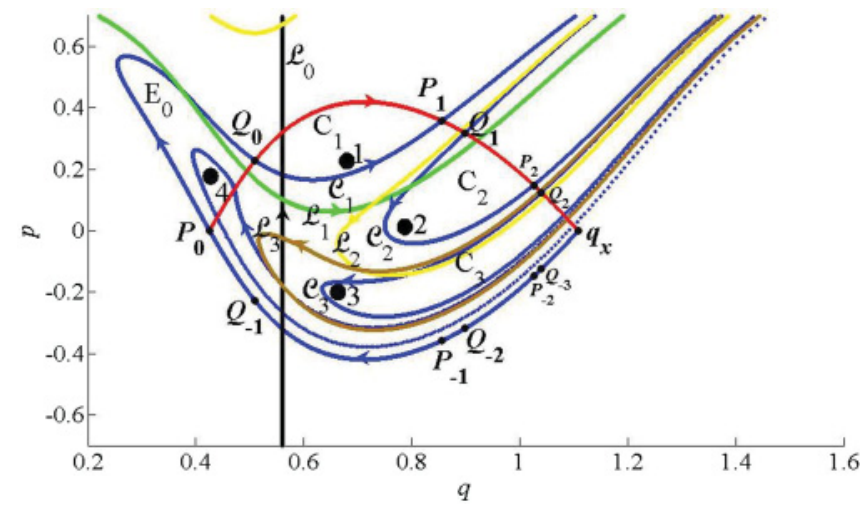

FIG. 3. (Color online) The same length of $\boldsymbol{u}$ [blue (gray)] plotted in Fig. 1(b) is shown. The vertical line segment (black) $\mathcal{L}_{0}=\left\{\left(q_{0}, p_{0}\right): q_{0}=0.5607,-1<p_{0}<1\right\}$, represents a burst of trajectories launched from the vase boundary point $(0.3,0.4067)$ (see Ref. [1]). $\mathcal{L}_{1}$ (green), $\mathcal{L}_{2}$ (yellow), and $\mathcal{L}_{3}$ (brown) are the first, second, and third images of $\mathcal{L}_{0}$, respectively.

With the development of the qualitative tangle in Fig. 2 and the evolution of the burst of trajectories in Figs. 3 and 4 , we have shown that the natural evolution of the tangle results in segments of curves that pass through $\mathrm{E}_{0}$, and thereby escape the complex. In the accompanying paper [1], we show that the number of iterates to escape the complex possesses a complicated recursive structure. Homotopic lobe dynamics (HLD) was invented to interpret and predict this recursive structure [23-29,31]. Below we give a brief review of HLD.

\section{HOMOTOPIC LOBE DYNAMICS}

We summarize homotopic lobe dynamics, a topological method that characterizes the dynamical transport of the tangle and allows us to predict sets of escape segments underlying the icicles in the continuous escape time plot presented in part I. Let us return to Fig. 2, and identify the two sets $\mathrm{H}_{1}=\mathrm{C}_{1} \cap \mathrm{E}_{-1}$ and $\mathrm{H}_{2}=\boldsymbol{M}\left(\mathrm{H}_{1}\right)=\mathrm{C}_{2} \cap \mathrm{E}_{0}$. The lobe $\mathrm{C}_{3}$ in Fig. 2(b) appears to wind around $\mathrm{H}_{1}$ and $\mathrm{H}_{2}$. Now call the set $\mathrm{H}_{3}=\mathrm{C}_{3} \cap \mathrm{E}_{1}$ and consider $\mathrm{C}_{4}$ in Fig. 2(c). $\mathrm{C}_{4}$ appears to wind around the three sets $\mathrm{H}_{1}, \mathrm{H}_{2}$, and $\mathrm{H}_{3}$ as if the lobe were avoiding these regions. Similarly, if we call the set $\mathrm{H}_{4}=\mathrm{C}_{4} \cap \mathrm{E}_{2}$, we can see that $\mathrm{C}_{5}$ winds around the four sets $\mathrm{H}_{1}, \mathrm{H}_{2}, \mathrm{H}_{3}$, and $\mathrm{H}_{4}$. In each case, the lobe appears to wind around the intersections of capture and escape lobes. HLD uses this observation to develop a topological dynamics describing how the boundaries of successive capture lobes wind through the plane.

\section{A. Defining holes}

We call $\boldsymbol{S}_{0}^{F}=\boldsymbol{S}\left[\boldsymbol{P}_{0}, \boldsymbol{P}_{1}\right)$ and $\boldsymbol{U}_{0}^{F}=\boldsymbol{U}\left[\boldsymbol{P}_{-1}, \boldsymbol{P}_{0}\right)$ the "fundamental $S$ and $\boldsymbol{U}$ segments." For any homoclinic point $\boldsymbol{x}$, let $m \in \mathbb{Z}$ be such that $\boldsymbol{M}^{m}(\boldsymbol{x}) \in \boldsymbol{U}_{0}^{F}$. The "transition number" $n$ is an integer such that $\mathcal{M}^{m+n}(\boldsymbol{x}) \in S_{0}^{F}$. In other words, the transition number is the number of iterates for a homoclinic point to map from $\boldsymbol{U}_{0}^{F}$ to $S_{0}^{F}$. In Fig. 1(b), the points $\left(\boldsymbol{\alpha}_{\boldsymbol{4}}, \boldsymbol{\beta}_{4}\right)$, which are the "corners of the set" $\mathrm{C}_{4} \cap \mathrm{E}_{0}$, have transition number 4 because the fourth preimage of each point lies in $\boldsymbol{U}_{0}^{F}$. Examining Fig. 1(a), we see that $\mathrm{C}_{4}$ intersects $\mathrm{E}_{-1}, \mathrm{E}_{-2}$, and $\mathrm{E}_{-3}$. This observation implies that $\mathrm{C}_{5}, \mathrm{C}_{6}$, and $\mathrm{C}_{7}$, are forced to intersect $\mathrm{E}_{0}$. The reader can verify that these homoclinic points will have transition numbers 5,6 , and 7 , respectively.

We will use the transition number together with the earlier observations of the shapes of successive capture lobes to alter the topology of the surface of section. Let $\boldsymbol{x}$ and $\boldsymbol{x}^{\prime}$ be homoclinic points. If $\boldsymbol{S}\left(\boldsymbol{x}, \boldsymbol{x}^{\prime}\right)$ and $\boldsymbol{U}\left(\boldsymbol{x}, \boldsymbol{x}^{\prime}\right)$ contain no additional homoclinic points, then $\boldsymbol{x}$ and $\boldsymbol{x}^{\prime}$ are said to be "neighbors." If we have a region bounded by segments of
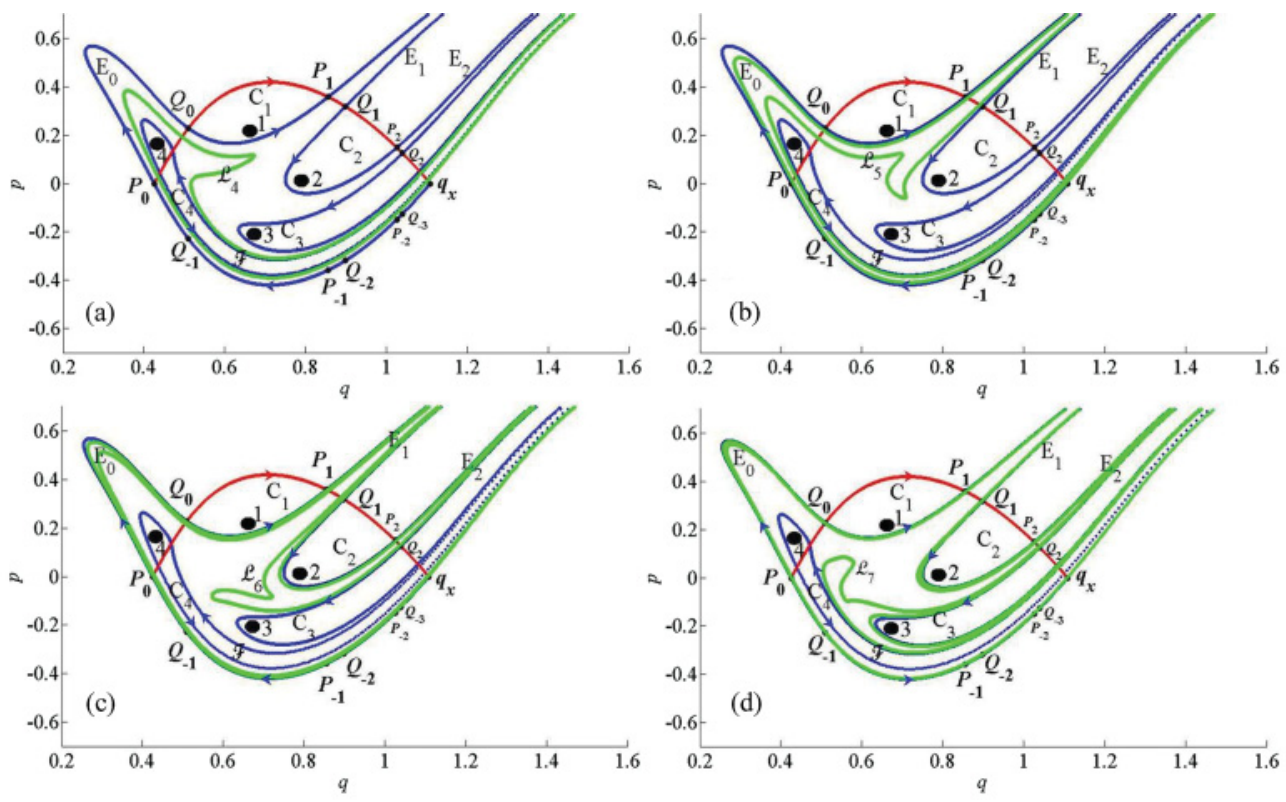

FIG. 4. (Color online) The (a) fourth $\left(\mathcal{L}_{4}\right)$, (b) fifth $\left(\mathcal{L}_{5}\right)$, (c) sixth $\left(\mathfrak{L}_{6}\right)$, and (d) seventh $\left(\mathcal{L}_{7}\right)$ iterates of the burst of trajectories $\left(\mathfrak{L}_{0}\right)$ shown in Fig. 3. 
$\boldsymbol{S}$ and $\boldsymbol{U}$ connecting a pair of neighbors, then by definition, the manifolds do not enter this region. However, in order to prove that two homoclinic points are neighbors, we need to know arbitrarily long segments of both $S$ and $\boldsymbol{u}$. Therefore we will use the following weaker but more pragmatic concept. Let $\boldsymbol{x}$ and $\boldsymbol{x}^{\prime}$ be homoclinic points. If $\boldsymbol{x}$ and $\boldsymbol{x}^{\prime}$ have transition number $\leqslant J$ and both $\boldsymbol{S}\left(\boldsymbol{x}, \boldsymbol{x}^{\prime}\right)$ and $\boldsymbol{U}\left(\boldsymbol{x}, \boldsymbol{x}^{\prime}\right)$ contain no homoclinic points of transition number $\leqslant J$, then $x$ and $\boldsymbol{x}^{\prime}$ are said to be "pseudoneighbors of index $J$ " or " $J$-neighbors."

The pseudoneighbors are found from computing $\cup_{i=0}^{J} \boldsymbol{u}_{i}^{F}=$ $\boldsymbol{u}$. In Fig. 1(b), we display the boundaries of capture lobes $\mathrm{C}_{1}, \ldots, \mathrm{C}_{4}$, so we may set $J=4$. We see that the points $\boldsymbol{\alpha}_{4}$ and $\boldsymbol{\beta}_{4}$ are 4-neighbors. Once we have identified pseudoneighbor pairs, we associate a hole with each pair. Let $\boldsymbol{x}$ and $\boldsymbol{x}^{\prime}$ be a pair of $J$-neighbors. We arbitrarily choose one of these points and anchor the hole to that point so that the hole lies in the region bounded by the two segments $\boldsymbol{S}\left(\boldsymbol{x}, \boldsymbol{x}^{\prime}\right)$ and $\boldsymbol{u}\left(\boldsymbol{x}, \boldsymbol{x}^{\prime}\right)$, and is infinitesimally close to the anchor. Mapping a hole forwards generates an infinite sequence of holes that eventually lie infinitesimally close to the boundary $\Gamma_{S}$, and that may or may not lie inside $\Gamma[27,32]$. After a finite number of iterates backwards, an anchor's preimage lands in $\boldsymbol{u}_{0}^{F}$ and thus the hole lies an infinitesimal distance from $\Gamma u$. The remaining preimages lie within an infinite sequence that asymptotically approaches $\boldsymbol{q}_{\boldsymbol{x}}$ (all lying an infinitesimal distance from $\Gamma \boldsymbol{u}$ that may or may not lie inside $\Gamma$ ).

In Fig. 1(b), we have placed disks representing the holes close to the actual holes. Each hole is identified by an integer. The hole disk 4 is placed near the homoclinic point $\boldsymbol{\alpha}_{\mathbf{4}}$. The images of disk 4 form a sequence of holes that all lie outside the complex, and that asymptotically approach $\boldsymbol{q}_{\boldsymbol{x}}$ near the stable boundary. The first three preimages, disk 3, disk 2, and disk 1, all lie inside the complex. The fourth preimage, disk 0 , lies outside the complex. All preimages of disk 0 lie outside the complex, and we can ignore these holes.

\section{B. Bridges and bridge classes}

The holes change the topology of the surface of section, and we can construct a symbolic representation of $\boldsymbol{U}$ that describes how it winds around the holes. For that purpose, we define a "homotopy class." If an oriented curve can be continuously deformed into another oriented curve without passing through a hole, we say that the two curves are "homotopic" [24,34]. A set of homotopic curves make up an equivalency class, and thus, any member of a homotopy class is a representative of the class. If $\mathfrak{B}$ denotes a curve, then its homotopy class is denoted as $[\mathscr{B}]$. We consider only curves and homotopy classes of curves whose end points are restricted to move along $\Gamma_{s}$ without leaving $\Gamma_{S}$.

Let us presume that we have numerically computed a finite segment of $\boldsymbol{u}$ called $\boldsymbol{u}$. After defining the holes, we assign specific segments of $\boldsymbol{u}$ to homotopy classes. A closed segment of $\boldsymbol{U}$ that intersects $\Gamma_{s}$ only at its end points is called a bridge. Two bridges can intersect only at their end points because $\boldsymbol{u}$ cannot self-intersect. $\boldsymbol{u}$ cuts across $\Gamma_{S}$ at multiple points so it can naturally be decomposed into a sequence of bridges. A homotopy class represented by a bridge is called a "bridge class." The orientation of a bridge class's representative is defined such that measured along $\Gamma_{s}$, the final point of the curve is closer to $\boldsymbol{q}_{\boldsymbol{x}}$ than the initial point. A bridge (and a bridge class) lying inside $\Gamma$ is said to be "internal" and lying outside $\Gamma$ is "external."

In Fig. 1(b), the oriented curves $\boldsymbol{e}_{1}, \boldsymbol{C}_{2}$, and $\boldsymbol{C}_{3}$, which are the unstable boundaries of $\mathrm{C}_{1}, \mathrm{C}_{2}$, and $\mathrm{C}_{3}$, are all bridges. Since each curve encloses a hole, each represents a unique bridge class (the inverse classes are obtained by traversing the curves backwards). The bridge $\boldsymbol{u}_{0}$ encloses disk 4 and represents a bridge class we denote as $u_{0}$. The bridge $\boldsymbol{u}_{1}$ encloses disk 5 and represents a bridge class we denote as $u_{1}$. The $n$th external escape lobe's unstable boundary is the bridge $\boldsymbol{u}_{n}$ and represents the bridge class $u_{n}$. Finally, we have the bridges $\mathcal{F}_{\text {and }} \mathcal{F}^{-1}$, which are two of the three bridges making up the unstable boundary of $\mathrm{C}_{4}$. Both bridges enclose the three internal holes, disk 1, disk 2, and disk 3.

Products of curves, such as $\boldsymbol{C}_{1} \boldsymbol{e}_{2}$, and products of homotopy classes, such as $\left[\boldsymbol{C}_{1}\right]\left[\boldsymbol{C}_{2}\right]$, are defined by traveling along the first curve $\boldsymbol{C}_{1}$, then, if necessary, traveling along the stable manifold from the final point of $\boldsymbol{C}_{1}$ to the initial point of $\boldsymbol{C}_{2}$, and finally traveling along $\boldsymbol{C}_{2}$ to its end point. With this definition, the reader can easily verify that $\mathcal{F}$ and $\mathcal{F}^{-1}$ are homotopic to the curves $\boldsymbol{C}_{1} \boldsymbol{e}_{2} \boldsymbol{e}_{3}$ and $\left(\boldsymbol{C}_{1} \boldsymbol{e}_{2} \boldsymbol{e}_{3}\right)^{-1}$, respectively and thus represent the classes $\left[\boldsymbol{C}_{1}\right]\left[\boldsymbol{C}_{2}\right]\left[\boldsymbol{C}_{3}\right]$ and $\left(\left[\boldsymbol{C}_{1}\right]\left[\boldsymbol{C}_{2}\right]\left[\boldsymbol{C}_{3}\right]\right)^{-1}$, respectively.

\section{Dynamical equations acting on the bridge classes}

Once all of the bridge classes have been identified, we use the dynamics of the plane $(\boldsymbol{M})$ to obtain dynamics on the set of bridge classes. We will also call this new mapping $\boldsymbol{M}$. First, let $c_{i}=\left[\boldsymbol{C}_{\boldsymbol{i}}\right]$ for $i=1,2,3$. Then, $\mathrm{C}_{2}=\boldsymbol{M}\left(\mathrm{C}_{1}\right)$ and $\mathrm{C}_{3}=\mathcal{M}\left(\mathrm{C}_{2}\right)$ induce the relationships $c_{2}=\mathcal{M}\left(c_{1}\right)$ and $c_{3}=\boldsymbol{M}\left(c_{2}\right)$. Likewise, $\boldsymbol{u}_{n}=\boldsymbol{M}^{n}\left(\boldsymbol{U}_{0}\right)$ induces the dynamical equation $u_{n}=\mathcal{M}^{n}\left(u_{0}\right)$. To obtain the dynamical equation for $c_{3}$, we must expand $\boldsymbol{C}_{4}$ in a sequence of bridge classes. Figure $1(\mathrm{~b})$ shows that $\left[\boldsymbol{C}_{4}\right]=\left[\mathcal{F}^{-1} \boldsymbol{U}_{0}^{-1} \mathfrak{F}\right]=\left[\mathcal{F}^{-1}\right]\left[\boldsymbol{U}_{0}^{-1}\right][\mathcal{F}]$. If we let $[\mathcal{F}]=f$, then we have the dynamical equation $\mathcal{M}\left(c_{3}\right)=f^{-1} u_{0}^{-1} f$. To obtain the dynamical equations for an inverse class, we use the relationship $\mathcal{M}\left(g^{-1}\right)=(\mathcal{M}(g))^{-1}$. Finally, we can classify bridge classes as either inert or active. A bridge class $u$ is said to be inert if $\boldsymbol{M}^{n}(u)$ is a bridge class $\forall n>0$ [32]. A bridge class that is not inert is said to be active.

Once we have identified the dynamical equations, we then construct the "bridge basis." An element in the bridge basis must directly enclose a finite number of holes with no other element directly enclosing those holes. We use the symbol " $a \triangleleft \mathrm{H}$ " to denote the phrase "the bridge class $a$ surrounds hole H." If $a$ is a bridge class and is an element of the bridge basis, then there is not a bridge class $b$ that satisfies the ordering $a \triangleleft b \triangleleft \mathrm{H}$. It follows from the definition that elements in the bridge basis are irreducible, i.e., they cannot be expressed as a product of other elements of the bridge basis. For our example, the symbols $c_{1}, c_{2}, c_{3}$, and $u_{n}$ for all $n$ (including inverses) make up the bridge basis. As we have stated, we use the first $J$ images of $\boldsymbol{u}_{0}^{F}$ to define holes that alter the topology of the plane. We call the corresponding bridge basis the "J-basis." If $\mathscr{B}$ is a curve, then the homotopy class it represents in the 

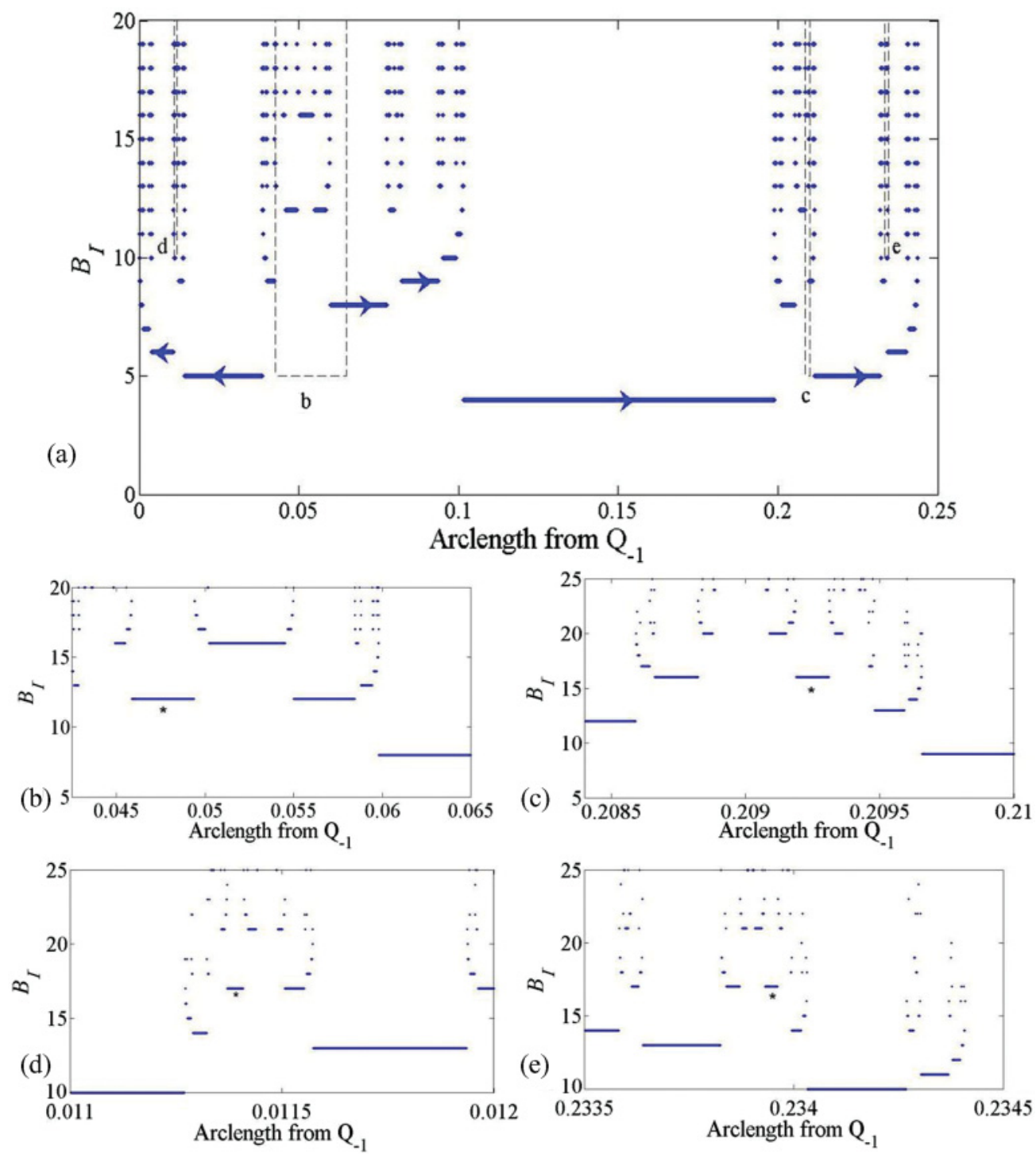

FIG. 5. (Color online) The numerically computed escape segments for $\boldsymbol{U}_{0}^{F}$. The horizontal axis represents the initial condition, which is the arc length measured along $\boldsymbol{U}$ starting from the homoclinic point $\boldsymbol{Q}_{-1}$. The vertical axis $\left(B_{\Gamma}\right)$ is the number of bounces to escape the complex. Here and in all of the following figures, an asterisk below an escape segment means that that escape segment is not a member of an epistrophe. (a) All escape segments for $\boldsymbol{u}_{0}^{F}$. Each outlined box encloses a specific subset of escape segments expanded in (b)-(e) and is discussed in the text. (b) Isolated escape segment at 12 iterates that corresponds to the finger in $\boldsymbol{C}_{12}$. (c) Isolated escape segment at 16 iterates corresponding to the finger in $\boldsymbol{C}_{16}$. (d) and (e) Isolated escape segments at iterate 17.

$J$-basis is denoted as $[\mathfrak{B}]_{J}$. We will use the term " $J$-dynamics" to mean the set of dynamical equations acting on the $J$-basis.

After identifying the bridge basis and the dynamical equations that act on the basis, the final step in the method is to put the dynamical equations into "concise form." A symbolic expression in concise form consists of a string of alternating internal and external symbols. When the dynamical equations are in concise form, symbolic expressions will also be in concise form. (Concise dynamical equations are used to compute a topological entropy, which we will discuss later.) In the present case, when we consistently use $f$ instead of the product $c_{1} c_{2} c_{3}$, symbolic expressions generated by the 4-dynamics will be in concise form.

Iteration of the symbolic dynamical equations in the $J$-basis predicts a minimal set of topologically forced escape segments for the curve of initial conditions $\boldsymbol{U}_{0}^{F}$. A second way to use the same symbolic equations is to apply them to other curves of initial conditions. In such applications, we are making use of the fact that other curves of initial conditions may approach $\boldsymbol{u}_{n}^{F}$ over a large part of their length (Figs. 3 and 4). Once the bridge basis has been identified, we can assign other curves to bridge classes within the $J$-basis, such as the images of $\mathcal{L}_{0}$ in Figs. 3 and 4 . The vertical line segment $\mathfrak{L}_{0}$ (black) represents a burst of trajectories. Since only one end point of $\mathcal{L}_{0}$ intersects $\Gamma_{S}$, the curve must be iterated once in order for both end points to intersect $\Gamma_{s}$. After one iterate, both end points intersect $\Gamma_{\mathcal{S}}$, and we see that the curve can be assigned to the bridge class $c_{1}$. Then iteration of the symbolic equations with initial condition $c_{1}$ produces a minimal set of escape segments for the burst of trajectories represented by $\mathcal{L}_{0}$. 


\section{Iteration of the dynamical equations in the 4-basis}

Summarizing the formulas derived in the preceding section, we have

$$
\begin{array}{llrl}
\mathcal{M}\left(c_{1}\right) & =c_{2}, & & \boldsymbol{M}\left(c_{2}\right)=c_{3}, \\
\mathcal{M}\left(c_{3}\right)=f^{-1} u_{0}^{-1} f, & & f=c_{1} c_{2} c_{3}, \\
\boldsymbol{M}(f)=c_{1}^{-1} u_{0}^{-1} f, & & \boldsymbol{M}^{n}\left(u_{0}\right)=u_{n} .
\end{array}
$$

Using Eq. (1), let us compute the homotopy classes of the first eight iterates of $\boldsymbol{u}_{0}^{F} \cdot \boldsymbol{u}_{0}^{F}$ cannot be assigned to a bridge class since only one end point lies in $\Gamma_{s}$, but its image $\boldsymbol{u}_{1}^{F}$ can be assigned to the class $u_{0} c_{1}$. We present the first eight iterates of $u_{0} c_{1}$ in Eq. (2):

$$
\begin{aligned}
{\left[\boldsymbol{u}_{1}^{F}\right]_{4}=} & \boldsymbol{u}_{0} c_{1}, \quad\left[\boldsymbol{u}_{2}^{F}\right]_{4}=u_{1} c_{2}, \\
{\left[\boldsymbol{u}_{3}^{F}\right]_{4}=} & u_{2} c_{3}, \quad\left[\boldsymbol{u}_{4}^{F}\right]_{4}=u_{3} f^{-1} \boldsymbol{u}_{0}^{-1} f, \\
{\left[\boldsymbol{u}_{5}^{F}\right]_{4}=} & u_{4} f^{-1} \boldsymbol{u}_{0} c_{1} u_{1}^{-1} c_{1}^{-1} \boldsymbol{u}_{0}^{-1} f, \\
{\left[\boldsymbol{u}_{6}^{F}\right]_{4}=} & u_{5} f^{-1} \boldsymbol{u}_{0} c_{1} u_{1} c_{2} u_{2}^{-1} c_{2}^{-1} u_{1}^{-1} c_{1}^{-1} \boldsymbol{u}_{0}^{-1} f, \\
& {\left[\boldsymbol{u}_{7}^{F}\right]_{4}=u_{6} f^{-1} \boldsymbol{u}_{0} c_{1} u_{1} c_{2} u_{2} c_{3} u_{3}^{-1} c_{3}^{-1} u_{2}^{-1} c_{2}^{-1} u_{1}^{-1} c_{1}^{-1} \boldsymbol{u}_{0}^{-1} f, } \\
& {\left[\boldsymbol{u}_{8}^{F}\right]_{4}=u_{7} f^{-1} \boldsymbol{u}_{0} c_{1} u_{1} c_{2} u_{2} c_{3} u_{3} f^{-1} \boldsymbol{u}_{0}^{-1} f u_{4}^{-1} f^{-1} \boldsymbol{u}_{0} f u_{3}^{-1} } \\
& \times c_{3}^{-1} u_{2}^{-1} c_{2}^{-1} u_{1}^{-1} c_{1}^{-1} \boldsymbol{u}_{0}^{-1} f .
\end{aligned}
$$

We are primarily interested in how $\boldsymbol{u}$ is forced to wind through $\mathrm{E}_{0}$. Each factor of $u_{j}^{ \pm 1}$ in $\left[\boldsymbol{u}_{n}^{F}\right]_{4}$ represents a segment that is predicted to escape at iterate $n-j$. A factor of $u_{j}^{-1}$ indicates that the segment is an element of an epistrophe that converges to the right, while each $u_{j}$ is an element of an epistrophe that converges to the left.

\section{E. Numerically computed escape segments}

Figure 5(a) shows the numerically computed set of escape segments of $\boldsymbol{U}_{0}^{F}$. The horizontal axis is the arc length measured along $\boldsymbol{U}$ starting at $\boldsymbol{Q}_{-1}$. An escape segment at $n$ iterates corresponds to a segment of $\boldsymbol{u}_{n}^{F}$ passing through $\mathrm{E}_{0}$. We see that there are two prominent epistrophes starting at four and five iterates with opposing orientations. If we drew arrows on each escape segment, then those in the rightward-converging epistrophe would each possess a rightward-pointing arrow. Similarly, each of the escape segments in the leftwardconverging epistrophe would possess a leftward-pointing arrow.

For the vertical line of initial conditions shown in Fig. 3, we show the numerically computed escape segments in Fig. 6. In Fig. 6, the large empty region in the center is where the line of initial conditions $\mathcal{L}_{0}$ overlaps the continent of stability in the surface of section [1]. The large escape segment at iterate 1 is where $\mathcal{L}_{0}$ overlaps escape lobe $\mathrm{E}_{-1}$. To compare Figs. 5 and 6, suppose we divide Fig. 5 into left-hand (LH) and right-hand (RH) portions on a line near arc length $=0.052$. Then (except for the segment at iterate 1 and the segment at iterate 11 marked by an asterisk in Fig. 6), up to iterate 11, every escape segment in the $\mathrm{LH}$ or $\mathrm{RH}$ portions of Fig. 5 corresponds to an escape segment in the $\mathrm{LH}$ or $\mathrm{RH}$ portion of Fig. 6. This is because (as shown in Figs. 3 and 4), iterates of $\mathcal{L}_{0}$ are close to iterates of $\boldsymbol{u}_{0}^{F}$.

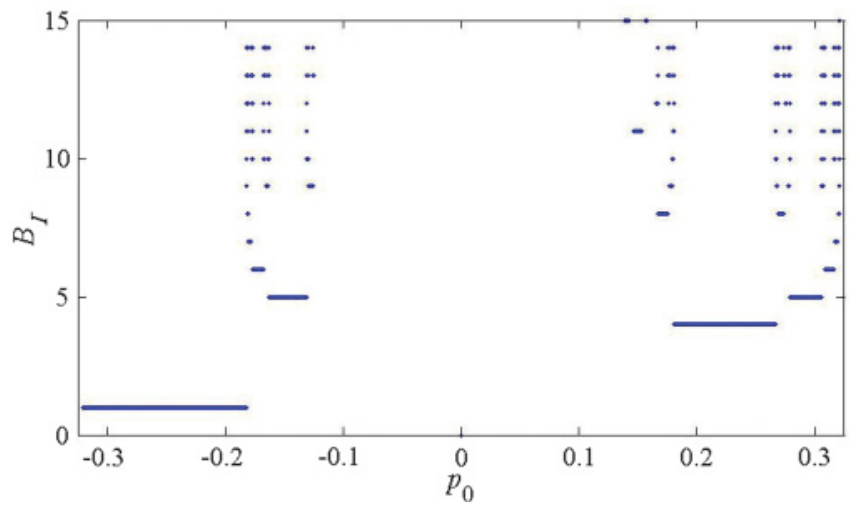

FIG. 6. (Color online) Escape segments for a burst of trajectories launched from the point $(0.3,0.4067)$. The horizontal axis is the initial condition; in this case it is the initial momentum (compare to Figs. 3 and 4).

\section{F. Comparison between topological prediction and numerical computation}

We compare Eq. (2), which contains the topologically forced set of escape segments at each iterate $\left(u_{0}^{ \pm 1}\right.$ in bold) to Fig. 5, which shows the numerically computed escape segments for $\boldsymbol{u}_{0}^{F}$. Scrutiny of the last expression in Eq. (2) and comparison with the figure indicates that there is complete agreement up to iterate 8 . Indeed, if we continue to iterate the symbolic expressions up to 11 iterates, we still find perfect agreement. However, on numerical computation of $\boldsymbol{u}_{12}^{F}$, we discover one additional escape segment [marked with an asterisk in Fig. 5(b)], not predicted by iteration of the 4-basis. Other unpredicted segments occur at $n=16$ and higher. This illustrates the fact that the symbolic dynamics predicts a minimal set of escape segments, and to get a more complete prediction, we need to examine higher-order basis sets.

\section{HIGHER-ORDER BASES}

The general epistrophe theorem [23] asserts that every escape segment has an epistrophe converging upon each of its end points. Therefore the additional escape segment at iterate 12 must spawn two epistrophes, and every escape segment in these two epistrophes must spawn two more epistrophes. To predict these by topological methods, we need to examine higher iterates of $\boldsymbol{u}_{0}^{F}$, choose holes, identify bridges, construct new bases, find symbolic dynamical equations, and apply them to generate improved predictions. For comparison to these higher bases, we have computed the number of escape segments appearing in $\left[\boldsymbol{u}_{i}^{F}\right]_{4}$ for $i=1,2, \ldots, 20$ and placed them in the second column of Table I. Higher bases always reproduce the results of the 4-basis, and may predict additional segments.

\section{A. The 12-basis}

This basis is constructed from the union of the first twelve iterates of $\boldsymbol{u}_{0}^{F}$. We find a "kink" in $\boldsymbol{u}_{12}^{F}$ resulting in two new pairs of 12-neighbors: $\left(\boldsymbol{\gamma}_{12}, \boldsymbol{\delta}_{12}\right)$ and $\left(\boldsymbol{\delta}_{\mathbf{1 2}}, \boldsymbol{\varepsilon}_{\mathbf{1 2}}\right)$ (Fig. 7). Accordingly, we define new holes, with the square hole associated with the pair $\left(\boldsymbol{\gamma}_{\mathbf{1 2}}, \boldsymbol{\delta}_{\mathbf{1 2}}\right)$, and the plus hole associated with the pair $\left(\boldsymbol{\delta}_{12}, \boldsymbol{\varepsilon}_{12}\right)$. The 4-neighbors $\left(\boldsymbol{\alpha}_{\mathbf{4}}, \boldsymbol{\beta}_{\mathbf{4}}\right)$ 
TABLE I. The first column contains the iterate number. The second, third, and fourth columns contain the number of escape segments predicted to appear in the first 20 iterates of $\left[\boldsymbol{U}_{0}^{F}\right]_{4},\left[\boldsymbol{U}_{0}^{F}\right]_{12}$, and $\left[\boldsymbol{U}_{0}^{F}\right]_{16}$, respectively. The sixth and seventh columns contain the same information for $\left[\mathcal{L}_{0}\right]_{4}$ and $\left[\mathcal{L}_{0}\right]_{16}$, respectively. And finally, the fifth and eighth columns contain the actual number of escape segments for $\boldsymbol{U}_{0}^{F}$ and $\mathcal{L}_{0}$, respectively.

\begin{tabular}{lccccccc}
\hline \hline$i$ & {$\left[\boldsymbol{U}_{i}^{F}\right]_{4}$} & {$\left[\boldsymbol{U}_{i}^{F}\right]_{12}$} & {$\left[\boldsymbol{U}_{i}^{F}\right]_{16}$} & $\boldsymbol{U}_{i}^{F}$ & {$\left[\mathcal{L}_{i}\right]_{4}$} & {$\left[\mathcal{L}_{i}\right]_{16}$} & $\mathcal{L}_{i}$ \\
\hline 1 & 0 & 0 & 0 & 0 & 0 & 0 & 0 \\
2 & 0 & 0 & 0 & 0 & 0 & 0 & 0 \\
3 & 0 & 0 & 0 & 0 & 0 & 0 & 0 \\
4 & 1 & 1 & 1 & 1 & 1 & 1 & 1 \\
5 & 2 & 2 & 2 & 2 & 2 & 2 & 2 \\
6 & 2 & 2 & 2 & 2 & 2 & 2 & 2 \\
7 & 2 & 2 & 2 & 2 & 2 & 2 & 2 \\
8 & 4 & 4 & 4 & 4 & 4 & 4 & 4 \\
9 & 8 & 8 & 8 & 8 & 8 & 8 & 8 \\
10 & 12 & 12 & 12 & 12 & 12 & 12 & 12 \\
11 & 16 & 16 & 16 & 16 & 16 & 16 & 17 \\
12 & 24 & 25 & 25 & 25 & 24 & 24 & 24 \\
13 & 40 & 40 & 40 & 40 & 40 & 40 & 40 \\
14 & 64 & 64 & 64 & 64 & 64 & 64 & 64 \\
15 & 96 & 96 & 96 & 96 & 96 & 96 & 98 \\
16 & 144 & 144 & 146 & 146 & 144 & 145 & 149 \\
17 & 224 & 224 & 228 & 228 & 224 & 226 & 228 \\
18 & 352 & 352 & 356 & 356 & 352 & 354 & 356 \\
19 & 544 & 544 & 548 & 548 & 544 & 546 & 552 \\
20 & 832 & 832 & 841 & 844 & 832 & 837 & 852 \\
\hline \hline
\end{tabular}

found from $\boldsymbol{u}_{4}^{F} \cap \mathrm{E}_{0}$ are also 12-neighbors and again a disk hole is associated with them.

The 12-dynamics is presented in detail in [35]. There are 31 symbols, and one might expect that they predict many more escape segments than the 4-dynamics. In fact, however, the predictions turn out to be identical to those obtained from the 4-dynamics, with the sole exception that there is one more segment in $\left[\boldsymbol{C}_{12}\right]_{12}$ (which is forced from construction). This is seen in Table I. We need to go to a 16-dynamics to significantly improve our predictions.

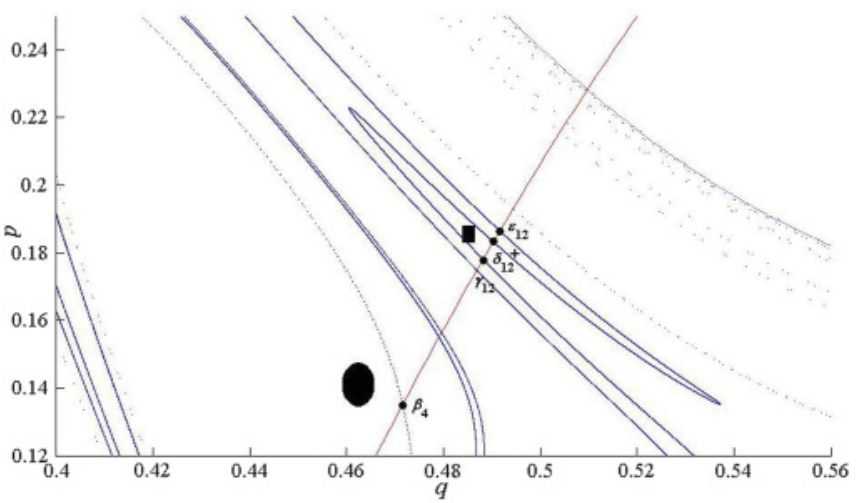

FIG. 7. (Color online) A kink in $\boldsymbol{U}_{12}^{F}$, the 12-neighbors, and the holes used to construct the 12-basis. The disk is placed near the hole attached to $\boldsymbol{\beta}_{\mathbf{4}}$. The square is attached to $\boldsymbol{\gamma}_{\mathbf{1 2}}$ and the plus is attached to $\delta_{12}$.

\section{B. The 16-basis}

\section{The 16-neighbors}

The 16-basis is derived from the union of the first 16 iterates of $\boldsymbol{u}_{0}^{F}$. Figure 8(a) shows the segments of $\boldsymbol{e}_{16}$ that pass through $\mathrm{E}_{0}$. Scrutinizing a graph of the 16 iterates of $\boldsymbol{u}_{0}^{F}$ gives the 16-neighbors. Since it would be impossible to display all 16 iterates, we elect to show a schematic representation in Fig. 8(b), which shows the segments of $\boldsymbol{U}$ connecting the three pairs of 16-neighbors and their associated holes. Each curve in Fig. 8(b) is representative of a segment of $\boldsymbol{U}$ either in $\boldsymbol{C}_{16}$ or $\boldsymbol{C}_{4}$. (We note that computing the 16-neighbors reveals that $\left(\gamma_{12}, \delta_{12}\right)$ and $\left(\delta_{12}, \varepsilon_{12}\right)$ are not true neighbors.) We find that the 16-neighbors $\left(\boldsymbol{\theta}_{\mathbf{1 6}}, \boldsymbol{l}_{\mathbf{1 6}}\right)$ are attached to a long tendril that is barely visible in Fig. 8(a) and encloses the plus hole. The pair $\left(\zeta_{\mathbf{1 6}}, \eta_{\mathbf{1 6}}\right)$ connect a segment of $\boldsymbol{U}$ that encloses the square hole. Finally, the 4-neighbors $\left(\boldsymbol{\alpha}_{\mathbf{4}}, \boldsymbol{\beta}_{\mathbf{4}}\right)$ are also 16-neighbors and again associated with a disk hole.

\section{The early minimal set generated by the 16-dynamics}

A schematic of the 16-basis is shown in Fig. 9 and the concise dynamical equations acting on the 16-basis are presented in Eqs. (3)-(38). We present a derivation of the dynamical equations in the Supplemental Material [36]. There are now three irreducible bridge classes in $\mathrm{E}_{0}$ that produce escape segments $\left(u_{0}, v_{0}\right.$, and $\left.t_{0}\right)$. The two possible sequences of successive escape classes are represented by $x_{0}$ and $y_{0}$, and the combination of all three is represented by $w_{0}$. (We note that in this basis, the path classes $c_{n}$ do not correspond to paths $\boldsymbol{C}_{n}$.)

$$
\begin{aligned}
& \boldsymbol{M}\left(a_{1}\right)=a_{2}, \\
& \boldsymbol{M}\left(b_{1}\right)=b_{2} \text {, } \\
& \boldsymbol{M}\left(c_{1}\right)=c_{2}, \\
& \mathcal{M}\left(d_{1}\right)=d_{2}, \\
& \boldsymbol{M}\left(f_{1}\right)=f_{2}, \\
& \boldsymbol{M}\left(a_{2}\right)=a_{3}, \\
& \mathcal{M}\left(b_{2}\right)=b_{3}, \\
& \boldsymbol{M}\left(c_{2}\right)=c_{3}, \\
& \boldsymbol{M}\left(d_{2}\right)=d_{3}, \\
& \boldsymbol{M}\left(f_{2}\right)=f_{3}, \\
& \boldsymbol{M}\left(a_{3}\right)=b_{4}^{-1} u_{0}^{-1} c_{4}, \\
& \boldsymbol{M}\left(b_{3}\right)=a_{4}^{-1} u_{0}^{-1} c_{4}, \\
& \boldsymbol{M}\left(c_{3}\right)=g^{-1} v_{0} f_{1} x_{0}^{-1} c_{4}, \\
& \boldsymbol{M}\left(d_{3}\right)=h^{-1} x_{0}^{-1} c_{4}, \\
& \boldsymbol{M}\left(f_{3}\right)=f_{4} \\
& \boldsymbol{M}\left(a_{4}\right)=c_{1}^{-1} w_{0}^{-1} c_{4}, \\
& \boldsymbol{M}\left(b_{4}\right)=b_{1}^{-1} w_{0}^{-1} c_{4}, \\
& \mathcal{M}\left(c_{4}\right)=a_{1}^{-1} w_{0}^{-1} c_{4}, \\
& \boldsymbol{M}\left(f_{4}\right)=c_{4}^{-1} t_{0}^{-1} c_{4}, \\
& \text { Let } g=f_{1} d_{1} f_{2} d_{2} f_{3} d_{3} f_{4} \text {, } \\
& \boldsymbol{M}(g)=d_{1}^{-1} w_{0}^{-1} c_{4}, \\
& \text { Let } h=d_{1} f_{2} d_{2} f_{3} d_{3} f_{4} \text {, }
\end{aligned}
$$




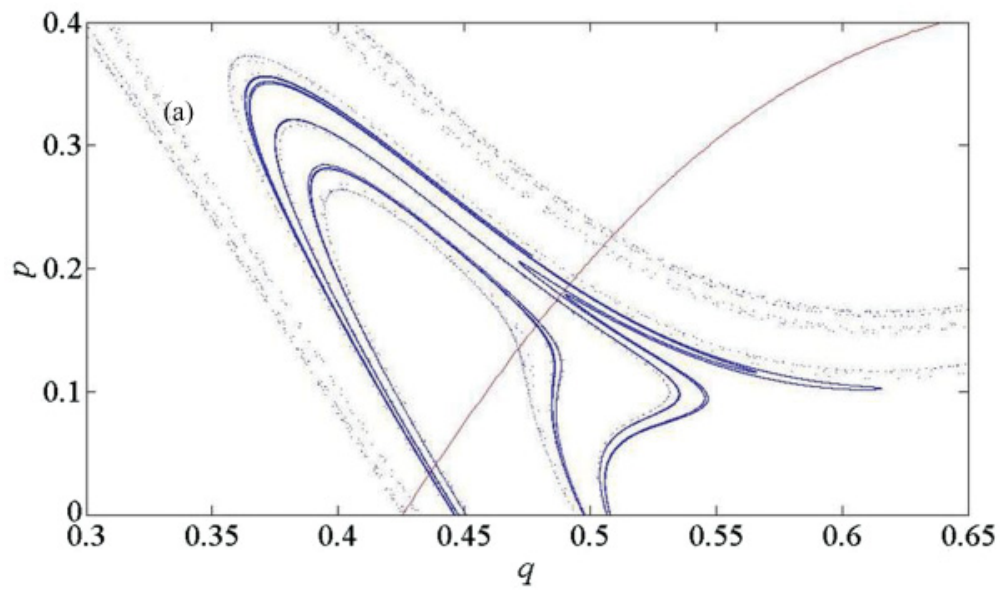

(b)

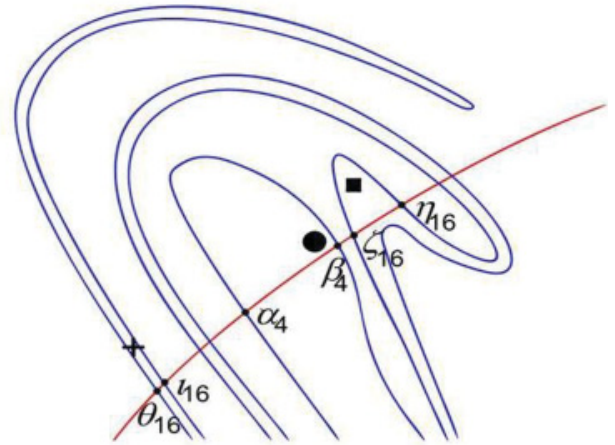

FIG. 8. (Color online) (a) The intersection of $\mathrm{C}_{16}$ with $\mathrm{E}_{0}$. (b) A schematic of (a) showing the three pairs of 16-neighbors and holes associated with each pair of 16-neighbors.

$$
\begin{gathered}
\boldsymbol{M}(h)=h_{1}^{-1} w_{0}^{-1} c_{4}, \\
h_{\mathrm{i}}=d_{i} f_{i+1}, \quad i=1,2,3, \\
\boldsymbol{M}\left(h_{1}\right)=h_{2}, \\
\boldsymbol{M}\left(h_{2}\right)=h_{3}, \\
\mathcal{M}\left(h_{3}\right)=h^{-1} w_{0}^{-1} c_{4}, \\
\boldsymbol{M}^{n}\left(t_{0}\right)=t_{n}, \\
\boldsymbol{M}^{n}\left(u_{0}\right)=u_{n}, \\
\boldsymbol{M}^{n}\left(v_{0}\right)=v_{n}, \\
\text { let } x_{0}=u_{0} v_{0}, \\
\boldsymbol{M}^{n}\left(x_{0}\right)=x_{n}, \\
y_{0}=t_{0} u_{0}, \\
\mathcal{M}^{n}\left(y_{0}\right)=y_{n}, \\
w_{0}=t_{0} u_{0} v_{0}, \\
\boldsymbol{M}^{n}\left(w_{0}\right)=w_{n} .
\end{gathered}
$$

First, let us examine the classes of the first eight iterates of $\boldsymbol{u}_{0}^{F}$ generated by the 16-dynamics in Eq. (39). Comparing the escape segments in Eq. (39) to those in Eq. (2), we see that the structures of the two minimal sets are identical up to the eighth iterate (though they are represented by different letters). In fact, the two minimal sets are identical up to the 11th iterate (see Table I). We find that the symbolic representation of $\left[\boldsymbol{U}_{16}^{F}\right]_{16}$ correctly predicts the set of escape segments in Fig. 5(a) up to 16 iterates, including the additional segments at 12 and 16 . (It must, since we constructed the symbolic dynamics from that information.) The interesting question is, compared to the 4-dynamics, how many additional escape segments the 16-dynamics predict past $\left[\boldsymbol{U}_{16}^{F}\right]_{16}$ ?

$$
\begin{aligned}
& {\left[\boldsymbol{u}_{1}^{F}\right]_{16}=\boldsymbol{u}_{0} a_{1}, \quad\left[\boldsymbol{u}_{2}^{F}\right]_{16}=u_{1} a_{2},} \\
& {\left[\boldsymbol{u}_{3}^{F}\right]_{16}=u_{2} a_{3}, \quad\left[\boldsymbol{u}_{4}^{F}\right]_{16}=u_{3} b_{4}^{-1} \boldsymbol{u}_{0}^{-1} c_{4},} \\
& {\left[\boldsymbol{u}_{5}^{F}\right]_{16}=u_{4} c_{4}^{-1} \boldsymbol{w}_{0} b_{1} u_{1}^{-1} a_{1}^{-1} \boldsymbol{w}_{0}^{-1} c_{4},} \\
& {\left[\boldsymbol{u}_{6}^{F}\right]_{16}=u_{5} c_{4}^{-1} \boldsymbol{w}_{0} a_{1} w_{1} b_{2} u_{2}^{-1} a_{2}^{-1} w_{1}^{-1} a_{1}^{-1} \boldsymbol{w}_{0}^{-1} c_{4},}
\end{aligned}
$$

$$
\begin{aligned}
{\left[\boldsymbol{u}_{7}^{F}\right]_{16}=} & u_{6} c_{4}^{-1} \boldsymbol{w}_{0} a_{1} w_{1} a_{2} w_{2} b_{3} u_{3}^{-1} a_{3}^{-1} w_{2}^{-1} a_{2}^{-1} \\
& \times w_{1}^{-1} a_{1}^{-1} \boldsymbol{w}_{0}^{-1} c_{4}, \\
{\left[\boldsymbol{u}_{8}^{F}\right]_{16}=} & u_{7} c_{4}^{-1} \boldsymbol{w}_{0} a_{1} w_{1} a_{2} w_{2} a_{3} w_{3} a_{4}^{-1} \boldsymbol{u}_{0}^{-1} c_{4} u_{4}^{-1} \\
& \times c_{4}^{-1} \boldsymbol{u}_{0} b_{4} w_{3}^{-1} a_{3}^{-1} w_{2}^{-1} a_{2}^{-1} w_{1}^{-1} a_{1}^{-1} \boldsymbol{w}_{0}^{-1} c_{4} .
\end{aligned}
$$

\section{Additional structure in the minimal set of $\mathcal{u}_{0}^{F}$ generated by the 16-dynamics}

(a) Structure associated with additional escape segment in $\boldsymbol{C}_{12}$. The bridge class $v_{0}$ goes around the square hole in Fig. 8(b). A comparable structure already appeared in $\boldsymbol{C}_{12}$; it is the bridge going around the square in Fig. 7, and its corresponding escape segment is the one with the asterisk in Fig. 5(b). We already mentioned that this segment must have epistrophes converging on each of its end points, and that those epistrophes are evident in Fig. 5(b), but only the sequence to the left of the isolated segment is predicted by the 12-dynamics. Happily, they are predicted by the 16-dynamics. If we generate 18 iterates in the 16-basis, then we find in $\left[\boldsymbol{U}_{18}^{F}\right]_{16}$ the sequence

$$
\begin{aligned}
{\left[\boldsymbol{u}_{18}^{F}\right]_{16}=} & u_{17} c_{4}^{-1} \cdots \boldsymbol{x}_{2}^{-1} a_{2}^{-1} \boldsymbol{w}_{1}^{-1} a_{1}^{-1} \boldsymbol{w}_{0}^{-1} c_{4} \boldsymbol{v}_{6} c_{4}^{-1} \boldsymbol{w}_{0} a_{1} \boldsymbol{w}_{1} a_{2} \\
& \times \boldsymbol{t}_{2}^{-1} a_{2}^{-1} \boldsymbol{w}_{1}^{-1} a_{1}^{-1} \boldsymbol{w}_{0}^{-1} c_{4} \boldsymbol{x}_{6}^{-1} c_{4}^{-1} \boldsymbol{w}_{0} a_{1} \boldsymbol{w}_{1} a_{2} \boldsymbol{u}_{2} \cdots c_{4} .
\end{aligned}
$$

The minimal set in Eq. (40) (in bold) is qualitatively plotted in Fig. 10. Comparing Figs. 10 and 5(b), we see that the 16-dynamics correctly predicts the appearance of epistrophes between $v_{6}$ and $x_{6}^{-1}$. More specifically, (i) $x_{6}^{-1}$ corresponds to the sixth iterate of the unasterisked segment at $B_{\Gamma}=12$ in Fig. 5(b); this segment is the first element of an epistrophe that was already predicted in the 4-basis. (ii) $v_{6}$ corresponds to the sixth iterate of the asterisked segment at $B_{\Gamma}=12$ in that figure; this is the new segment resulting from the kink in $\boldsymbol{C}_{12}$. (iii) The epistrophes converging upon these segments are correctly predicted in the 16-basis. The symbolic dynamics says that $v_{6}$ spawns its right-pointing epistrophe after four iterates and its left-pointing epistrophe after five iterates. The symbol $t_{2}^{-1}$ corresponds to the second iterate of the 


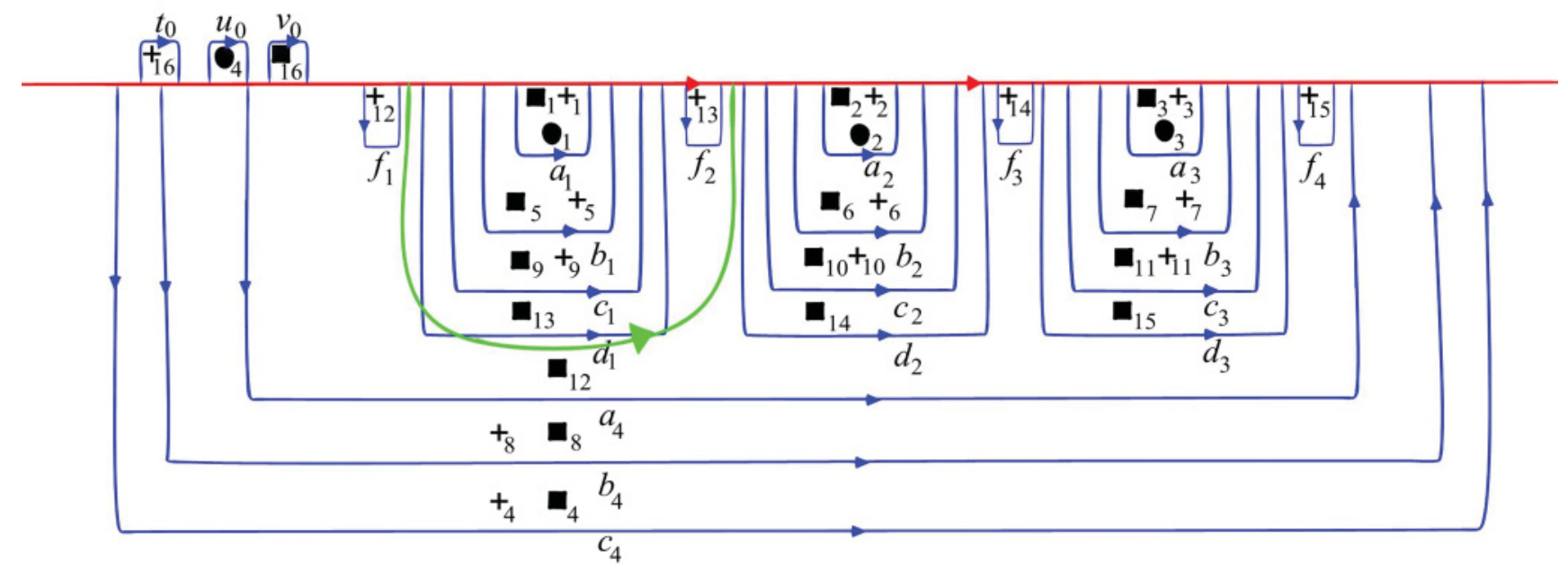

FIG. 9. (Color online) Schematic of the 16-basis. The curve enclosing the "1" bridge basis elements (green) is homotopic to $\mathcal{L}_{1}$ in Fig. 3 .

escape segment in Fig. 5(b) that appears to be shared by two epistrophes.

(b) Structure spawned by a segment in $\boldsymbol{C}_{16}$. In Fig. 5(c) an asterisk marks an isolated segment corresponding to a kink or finger in $\boldsymbol{C}_{16}$. A pair of epistrophes converging upon its end points appears to begin at $B_{\Gamma}=20$. Scrutiny of $\left[\boldsymbol{u}_{16}^{F}\right]_{16}$ indicates that the asterisked segment is represented by the bridge class $v_{0}^{-1}$. Continuing the iteration up to $B_{\Gamma}=21$, the same segment is represented by $v_{5}^{-1}$, and the symbolic representation nearby is

$$
\begin{aligned}
{\left[\boldsymbol{u}_{21}^{\mathrm{F}}\right]_{16}=} & \boldsymbol{u}_{20} c_{4}^{-1} \cdots \boldsymbol{u}_{1}^{-1} a_{1}^{-1} \boldsymbol{w}_{0}^{-1} c_{4} \boldsymbol{x}_{5} c_{4}^{-1} \boldsymbol{w}_{0} a_{1} \boldsymbol{t}_{1} a_{1}^{-1} \\
& \times \boldsymbol{w}_{0}^{-1} c_{4} \boldsymbol{v}_{5}^{-1} c_{4}^{-1} \boldsymbol{w}_{0} a_{1} \boldsymbol{x}_{1} \cdots c_{4}
\end{aligned}
$$

We see in Fig. 5(c) that the finger spawns an epistrophe on each side at iterate 20 . However, our calculation misses one escape segment. To the right of the bridge class $v_{5}^{-1}$, we see factors of $w_{0}$ and $x_{1}$, which correspond to the leftward-pointing escape segments spawned by the finger at iterates 21 and 20 , respectively. On the left, the factor of $w_{0}^{-1}$ corresponds to the rightward-pointing escape segment spawned at 21 iterates. However, the factor of $t_{1}$ corresponds to a leftward-pointing escape segment that is not spawned by the factor of $v_{5}^{-1}\left(t_{1}\right.$ is spawned by $x_{5}$ ). This means that our calculation did not

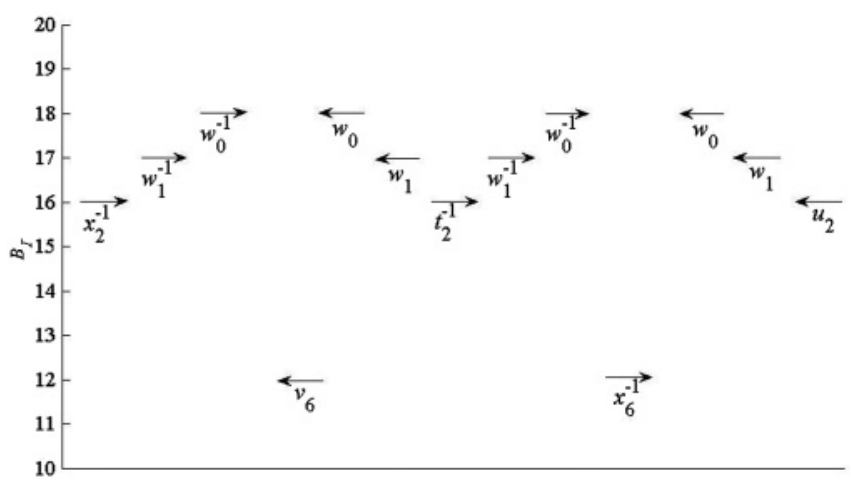

FIG. 10. (Color online) Schematic representation of the escape segments highlighted in Eq. (40). predict the rightward-pointing epistrophe spawned by the kink at iterate 20 .

(c) Structure spawned by two isolated segments in $\boldsymbol{C}_{17}$. We find that $\left[\boldsymbol{U}_{17}^{F}\right]_{16}$ contains two additional segments compared to $\left[\boldsymbol{u}_{17}^{F}\right]_{4}$, namely the symbols $v_{0}$ and $v_{0}^{-1}$. These are isolated escape segments and their locations in the escape time plot are shown in Figs. 5(d) and 5(e). We see that each isolated segment spawns two epistrophes at 21 iterates. We compare these results to Eq. (42), which shows that $\left[\boldsymbol{u}_{21}^{F}\right]_{16}$ contains factors of $v_{4}$ and $v_{4}^{-1}$ that represent the fourth iterates of the isolated segments in Figs. 5(d) and 5(e), respectively. To the left of $v_{4}$, we find a factor of $x_{0}^{-1}$, which is a rightward-pointing escape class spawned by $v_{4}$. We expect to find a leftward-pointing escape class immediately to the right of $v_{4}$ representing a class converging to the right end point of $v_{4}$. However, Eq. (42) shows that we find the rightward-pointing class $t_{0}^{-1}$ immediately to the right of $v_{4}$. This class is the first element of an epistrophe converging to the left end point of the class $x_{4}^{-1}$. We expect that the factor of $v_{4}^{-1}$ will also spawn two epistrophes on either side. However, we find that it only spawns one epistrophe which begins with the class $x_{0}$. The factor of $t_{0}$ to the immediate left of $v_{4}^{-1}$ belongs to an epistrophe converging to the right end point of $x_{4}$ :

$$
\begin{aligned}
{\left[\boldsymbol{u}_{21}^{F}\right]_{16} } & \\
= & u_{20} c_{4}^{-1} \cdots \boldsymbol{w}_{1} a_{2} \boldsymbol{w}_{2} a_{3} \boldsymbol{w}_{3} h^{-1} \boldsymbol{x}_{0}^{-1} c_{4} \boldsymbol{v}_{4} c_{4}^{-1} \boldsymbol{t}_{0}^{-1} c_{4} \boldsymbol{x}_{4}^{-1} c_{4}^{-1} \\
& \times \boldsymbol{u}_{0} b_{4} \boldsymbol{w}_{3}^{-1} \cdots \boldsymbol{w}_{1} a_{2} \boldsymbol{w}_{2} a_{3} \boldsymbol{w}_{3} b_{4}^{-1} \boldsymbol{u}_{0}^{-1} c_{4} \boldsymbol{x}_{4} c_{4}^{-1} \boldsymbol{t}_{0} c_{4} \boldsymbol{v}_{4}^{-1} \\
& \times c_{4}^{-1} \boldsymbol{x}_{0} h \boldsymbol{w}_{3}^{-1} \cdots c_{4} .
\end{aligned}
$$

\section{Summary of minimal sets predicted by the 4-, 12-, and 16-dynamics}

Table I summarizes our results. We see that the 4-dynamics and 12-dynamics give almost identical results; they differ in only one escape segment, which itself results directly from computing $\left[\boldsymbol{u}_{12}^{F}\right]_{12}$. We see that the 16 -dynamics itself recovers the additional segments at 12 and 16 iterates (again, by construction), and also predicts new structure past the 16th iterate. Up to the twentieth iterate, the 16-dynamics predicts only a 
few additional escape segments compared to the other two bases. Beyond the twentieth iterate, the 16-dynamics predicts more segments than the other bases, however, it still misses some segments that are found in our numerical calculations.

\section{Minimal sets for $\mathcal{L}_{0}$}

Let us now examine the predicted minimal set for the burst of trajectories generated by the 4- and 16-dynamics. We first expand $\left[\mathcal{L}_{1}\right]$ in each basis. Figure 3 shows that $\left[\mathcal{L}_{1}\right]_{4}=c_{1}$. Thus, the minimal set for $\mathcal{L}_{0}$ generated by the 4-dynamics is identical to that of $\boldsymbol{u}_{0}^{F}$.

Going to the 16-basis, Fig. 9 shows a curve homotopic to $\mathcal{L}_{1}$, from which it follows that $\left[\mathcal{L}_{1}\right]_{16}=d_{1} f_{2}$ or $h_{1}$ in concise form. We iterate this forward eight times to obtain Eq. (43). The last column in Table I shows the actual number of escape segments that appear at each iterate. We see from Eq. (43) that the 16-dynamics correctly predicts the number of escape segments up to eight iterates, and from Table I that it predicts the correct number of escape segments up to 10 iterates.

$$
\begin{aligned}
{\left[\mathcal{L}_{1}\right]_{16}=} & h_{1}, \quad\left[\mathcal{L}_{2}\right]_{16}=h_{2}, \\
{\left[\mathcal{L}_{3}\right]_{16}=} & h_{3}, \quad\left[\mathcal{L}_{4}\right]_{16}=h^{-1} \boldsymbol{w}_{0}^{-1} c_{4}, \\
{\left[\mathcal{L}_{5}\right]_{16}=} & c_{4}^{-1} \boldsymbol{w}_{0} h_{1} w_{1}^{-1} a_{1}^{-1} w_{1}^{-1} a_{1}^{-1} \boldsymbol{w}_{0}^{-1} c_{4}, \\
{\left[\mathcal{L}_{6}\right]_{16}=} & c_{4}^{-1} \boldsymbol{w}_{0} a_{1} w_{1} h_{2} w_{2}^{-1} a_{2}^{-1} w_{1}^{-1} a_{1}^{-1} \boldsymbol{w}_{0}^{-1} c_{4}, \\
{\left[\mathcal{L}_{7}\right]_{16}=} & c_{4}^{-1} \boldsymbol{w}_{0} a_{1} w_{1} a_{2} w_{2} h_{3} w_{3}^{-1} a_{3}^{-1} w_{2}^{-1} a_{2}^{-1} \\
& \times w_{1}^{-1} a_{1}^{-1} \boldsymbol{w}_{0}^{-1} c_{4}, \\
{\left[\mathcal{L}_{8}\right]_{16}=} & c_{4}^{-1} \boldsymbol{w}_{0} a_{1} w_{1} a_{2} w_{2} a_{3} w_{3} h^{-1} \boldsymbol{w}_{0}^{-1} c_{4} w_{4}^{-1} \\
& \times c_{4}^{-1} \boldsymbol{u}_{0} b_{4} w_{3}^{-1} a_{3}^{-1} w_{2}^{-1} a_{2}^{-1} w_{1}^{-1} a_{1}^{-1} \boldsymbol{w}_{0}^{-1} c_{4} .
\end{aligned}
$$

Figure 6 shows that the first isolated escape segment appears at 11 iterates near $p_{0} \approx 0.15$. We find that neither the 4-dynamics nor the 16-dynamics predict this isolated escape segment. This segment is not topologically forced by either the 4- or 16-dynamics. To understand why it appears, it is easiest to iterate backwards and examine the intersection of $\boldsymbol{L}_{0}$ and $\mathrm{E}_{-11}$ (Fig. 11). We see that a small finger in $\mathrm{E}_{-11}$ 's stable

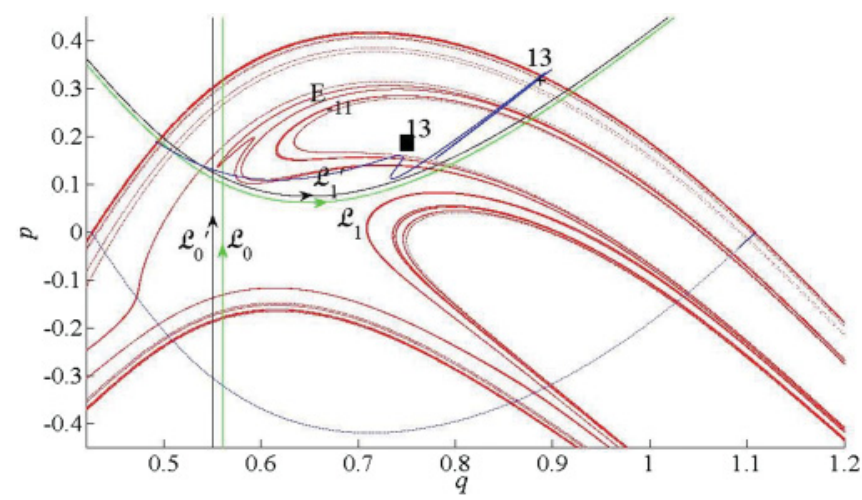

FIG. 11. (Color online) The isolated escape segment at 11 iterates in Fig. 6 results from an intersection of $\mathcal{L}_{0}$ (green) with the stable boundary of $\mathrm{E}_{-11}$ (red). The curve $\mathcal{L}_{1}$ (green) is the image of $\mathcal{L}_{0}$. The vertical line segment $\mathcal{L}_{0}^{\prime}$ (black) is a burst of trajectories with the initial position perturbed enough to eliminate the intersection of $\mathcal{L}_{0}$ and $\mathrm{E}_{-11}$. The curve $\mathcal{L}_{1}^{\prime}$ (black) is the image of the perturbed burst.

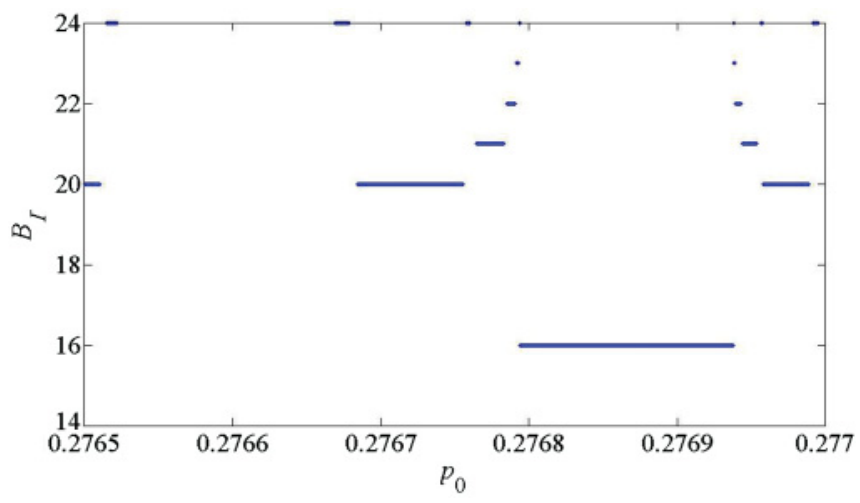

FIG. 12. (Color online) Isolated escape segment centered near $p_{0}=0.27685$.

boundary intersects $\mathcal{L}_{0}$ [green (gray) line segment]. The other vertical line segment $\mathcal{L}_{0}^{\prime}$ (black) is a small shift of $\mathcal{L}_{0}$, which does not intersect $\mathrm{E}_{-11}$. The curves $\mathcal{L}_{1}$ and $\mathcal{L}_{1}^{\prime}$ [green (gray) and black, respectively] are the first images of the vertical line segments. We see that both $\mathcal{L}_{1}$ and $\mathcal{L}_{1}^{\prime}$ are assigned to the same homotopy class, so their minimal sets are identical. Therefore, the isolated escape segment at 11 iterates cannot be predicted by the 16-dynamics.

Let us examine an escape segment predicted by the 16-basis that is not predicted by the 4-basis. The escape time plot near this isolated escape segment is shown in Fig. 12. We see that in actuality, this segment spawns two epistrophes at 20 iterates. We compare Fig. 12 to Eq. (44), which contains a substring of $\left[\mathcal{L}_{21}\right]_{16}$ :

$\left[\mathcal{L}_{21}\right]_{16}=c_{4}^{-1} \cdots \boldsymbol{x}_{5} c_{4}^{-1} \boldsymbol{w}_{0} a_{1} \boldsymbol{t}_{1} a_{1}^{-1} \boldsymbol{w}_{0}^{-1} c_{4} \boldsymbol{v}_{5}^{-1} c_{4}^{-1} \boldsymbol{w}_{0} a_{1} \boldsymbol{x}_{1} \cdots c_{4}$.

The isolated segment is predicted and is represented by the class $v_{5}^{-1}$. To the left of $v_{5}^{-1}$, we see the two escape classes $t_{1}$ and $w_{0}^{-1}$. The factor of $w_{0}^{-1}$ is the first element of an epistrophe that converges to the left end point of $v_{5}^{-1}$ and appears five iterates later. The factor of $t_{1}$ belongs to an epistrophe converging to the right side of $x_{5}$. The factors of $w_{0}$ and $x_{1}$ are members of an epistrophe converging to the right side of $v_{5}^{-1}$ which started four iterates after the appearance of $v_{0}^{-1}$. Therefore, we have the same situation demonstrated in Eq. (42), where the 16-dynamics predicted the $v_{0}$ class to spawn one epistrophe after four iterates and the second after five iterates.

The results for iterates of $\left[\mathcal{L}_{0}\right]_{J}$ are summarized in the last three columns of Table I. The 4-dynamics produces a minimal set that is identical to that for $\boldsymbol{U}_{0}^{F}$. Up to the fifteenth iterate, the 4 and 16-dynamics predict identical minimal sets. Past the fifteenth iterate, the 16-dynamics predicts additional escape segments. Specifically, the 16-dynamics predicts an additional segment at iterate 16, two additional segments at iterates 17-19, and five additional segments at iterate 20 .

The last column in Table I shows the number of computed escape segments in the first 20 iterates of $\boldsymbol{L}_{0}$. As we previously stated, the 16-dynamics is unable to predict the single isolated escape segment that appears at the eleventh iterate. Therefore, the 16-dynamics cannot account for the spawn of this isolated segment, two of which appear at iterates 15 through 18, six of which appear at iterate 19, and 10 of which appear at iterate 
20. Examining the last rows of the last column in Table I shows that there are even more segments unaccounted for by the 16-basis.

\section{REPRESENTATIONS OF COMPLEXITY}

We can compare the complexity of the 4-dynamics with that of the 16-dynamics by computing their topological entropies and by examining graphical representations of the two sets of dynamical equations.

\section{A. Topological entropy}

The definition of topological entropy depends on the context. We use it as a measure of the rate of increase of escape segments with increasing iterate [27]. To compute it, we first construct a "transition matrix." This is a matrix of integers where each element represents the number of symbols of each type produced upon applying the mapping to an element in the bridge basis. The topological entropy is defined to be the natural logarithm of the largest eigenvalue of the transition matrix. An increase in the topological entropy reflects an increase in the number of ways curves can be folded and stretched in the phase plane.

The transition matrix for the 4-dynamics is given in Eq. (45). To see how it is constructed, we return to Eq. (1). The symbol $u^{*}$ represents the whole set $\left\{u_{n}\right\}_{n=0}^{\infty}$, and the column underneath contains a single nonzero entry, which is the row labeled $u^{*}$. This means that $u_{n}$ maps into $u_{n+1}$, and can never produce anything else. Returning to Eq. (1), mapping $c_{1}$ and $c_{2}$ forward produces the symbols $c_{2}$ and $c_{3}$, respectively. Therefore, in Eq. (45), the column below the symbol $c_{1}$ contains a 1 in the $c_{2}$ row, and the column below $c_{2}$ has a 1 in the $c_{3}$ row. Finally, $\boldsymbol{M}\left(c_{3}\right)=f^{-1} u_{0}^{-1} f$ contains two factors of $f$ and a factor of $u^{*}$. Therefore, in column $f$ we place a 2 in row $f$ and a 1 in row $u^{*}$.

\begin{tabular}{|c|c|c|c|c|c|}
\hline & $c_{1}$ & $c_{2}$ & $c_{3}$ & $f$ & $u^{*}$ \\
\hline$c_{1}$ & 10 & 0 & 0 & 1 & $0)$ \\
\hline$c_{2}$ & 1 & 0 & 0 & 0 & 0 \\
\hline$c_{3}$ & 0 & 1 & 0 & 0 & 0 \\
\hline$f$ & 0 & 0 & 2 & 1 & 0 \\
\hline$u^{*}$ & 10 & 0 & 1 & 1 & $1)$ \\
\hline
\end{tabular}

The topological entropy for this matrix is $\ln (1.5437)$. For comparison, the topological entropy for a tangle with minimum delay time of 1 is $\ln (2)$. The lower topological entropy for our 4-dynamics reflects the larger minimum time delay, which leads to a longer delay between the appearance of an escape segment and the appearance of its progeny.

The topological entropy of the 12-dynamics is also $\ln (1.5437)$, because, although the 12-basis is much larger, its high-iterate predictions are identical to those of the 4-basis. The topological entropy of the 16-dynamics turns out to be $\ln (1.5449)$, which is slightly larger than the entropy for the dynamical equations acting on the 4-basis. Thus, the 16-basis encodes more complexity than the 4 - and 12-bases.

\section{B. Graphical representations of the dynamical equations}

For each set of dynamical equations, we constructed a graph that qualitatively encapsulates the dynamics. Each arrow represents a nonzero element of the transition matrix. In

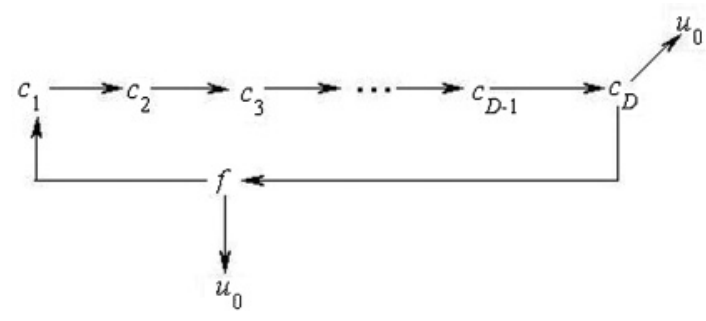

FIG. 13. (Color online) Graph qualitatively representing the dynamical equations acting on a $D$-basis for a system with minimum delay time $D$.

the graphs we do not include circular arrows indicating that a symbol produces a factor of itself, and we do not show multiple arrows if a symbol produces more than one of a given symbol. (Also, as in the transition matrix, we do not distinguish between elements and their inverses, and we collect all inert symbols into a single symbol.) The graphs therefore carry less information than the transition matrix, but they give a visual representation of how escape classes are repetitively generated.

First we consider a simple example, a tangle with minimum delay time $D$. The intersection of $\mathrm{C}_{D+1}$ with $\mathrm{E}_{0}$ produces a pair of $(D+1)$-neighbors. The dynamical equations are given in Eq. (46) and their derivation can be found in [27]:

$$
\begin{aligned}
\mathcal{M}\left(c_{i}\right) & =c_{i+1} \text { for } 0<i<D-1, \\
\mathcal{M}\left(c_{D}\right) & =f^{-1} u_{0}^{-1} f \text { where } f=c_{1} c_{2} \cdots c_{D}, \\
\mathcal{M}(f) & =c_{1}^{-1} u_{0}^{-1} f .
\end{aligned}
$$

Figure 13 shows the graph for Eq. (46). We see that if we start at any active symbol in the $D$-basis, we return to it $D$ +1 iterates later. Completing this circuit once generates two escape segments.

Figure 14 shows the graph for the 16-basis. The reader can verify that by starting at any active symbol, the map permits a path leading to every node in the graph, including the initial node. One may start with $a_{1}$, which is represented by $\boldsymbol{C}_{1}$, in the

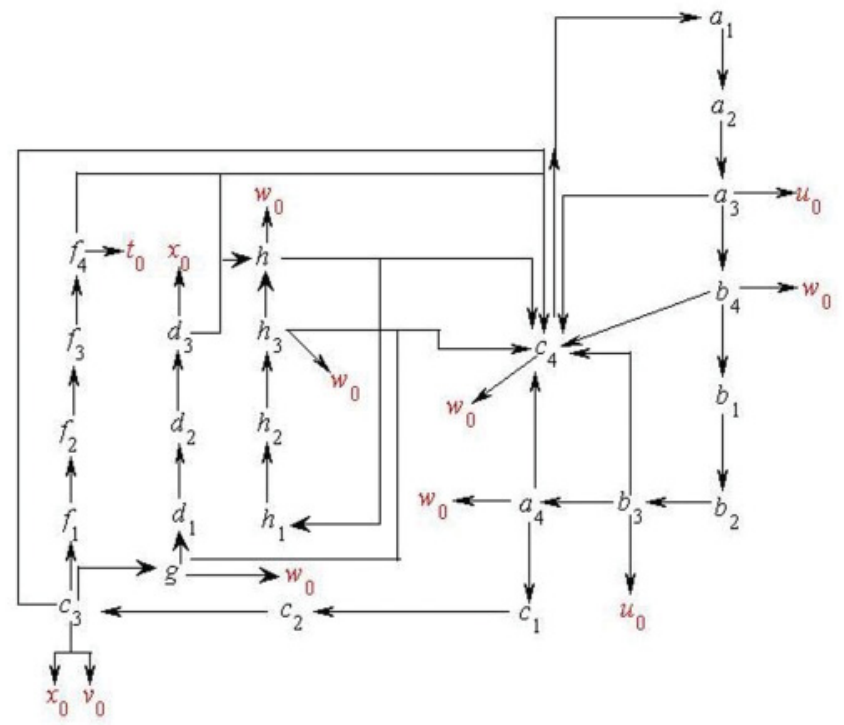

FIG. 14. (Color online) Graph qualitatively representing the dynamical equations acting on the 16-basis. 
upper right-hand corner. We invite the reader to wander around the various circuits in this graph to see how escape segments $t_{0}$, $u_{0}, v_{0}, w_{0}, x_{0}$, and $y_{0}$ are repeatedly produced. (We also invite the reader to see Fig. 4.6 of [35], which is the corresponding graph for the 12-basis. Here, the reader can verify that there is only one circuit containing the escape class representing the finger in $\boldsymbol{C}_{12}$ and that this circuit is not closed.)

\section{CONCLUSIONS}

We have presented a topological analysis of a homoclinic tangle generated from a chaotic, open, specularly reflecting vase-shaped cavity. We have applied the improved form of homotopic lobe dynamics that was presented in Ref. [27]. This method uses as input a calculation of a specified number of iterates $(J)$ of a fundamental segment of the unstable manifold. For three values of $J$, the method was applied to the unstable manifold itself and to a line of initial conditions representing a family of trajectories emanating from a single point on the boundary of the vase.

We applied the method for $J=4,12$, and 16 . We found that the 4-dynamics generated a minimal set that matches the earliest numerically computed epistrophes of $\boldsymbol{u}_{0}^{F}$. We saw that the minimal set for $\boldsymbol{u}_{0}^{F}$ generated by the 12 -basis is identical (except for a single escape segment) to that of the 4-basis. The 16-dynamics predicted more escape segments, indeed it predicted all the escape segments in $\boldsymbol{u}_{n}^{F}$ up to iterate 19 . We then examined the minimal sets for $\mathcal{L}_{0}$ generated by the 4 - and 16 dynamics. We saw that the 4-dynamics predicted the same minimal sets for $\mathcal{L}_{0}$ and $\boldsymbol{U}_{0}^{F}$. The 16-dynamics predicts several additional segments in $\mathcal{L}_{16}$ through $\mathcal{L}_{20}$, but it still does not predict all of the segments that are found in numerical computations.

Finally, we calculated the topological entropies of the 4-, 12-, and 16-dynamics. We found that the entropy of the 12-dynamics is the same as that of the 4-dynamics, but the entropy of the 16-dynamics is larger. These results are all consistent with the calculations and observations presented in the accompanying paper.

We should remark that while the various versions of homotopic lobe dynamics efficiently predict the overwhelming majority of the escape segments that occur at short to intermediate times, "surprises"-that is, unpredicted segmentscontinue to occur at late times, and therefore at all levels of resolution. The unpredicted segments are often large, and they may come to dominate at long times.

\section{ACKNOWLEDGMENT}

This work was supported by the National Science Foundation (NSF).
[1] J. A. Novick and J. B. Delos, Phys. Rev. E 85, 016205 (2012).

[2] R. W. Easton, Transactions of the American Mathematical Society 294, 719 (1986).

[3] R. W. Easton, Geometric Methods for Discrete Dynamical Systems (Oxford University Press, New York, 1998).

[4] B. Eckhardt, J. Phys. A 20, 5971 (1987).

[5] J. Meiss, Rev. Mod. Phys. 64, 795 (1992).

[6] V. Rom-Kedar, Physica D 43, 229 (1990).

[7] V. Rom-Kedar, Chaos 5, 385 (1995).

[8] V. Rom-Kedar, A. Leonard, and S. Wiggins, J. Fluid Mech. Digital Archive 214, 347 (2006).

[9] P. Collins, in Geometry and Topology in Dynamics: AMS Special Session on Topology in Dynamics held in Winston-Salem, NC, October 9-10, 1998; AMS-AWM Special Session on Geometry in Dynamics held in San Antonio, TX, January 13-16, 1999, edited by M. Barge and K. Kuperberg, Contemporary Mathematics, Vol. 246 (American Mathematical Society, Providence, RI, 1999), p. 65.

[10] P. Collins and B. Krauskopf, Phys. Rev. E 66, 56201 (2002).

[11] P. Collins, Int. J. Bifurcation Chaos Appl. Sci. Eng. 12, 605 (2002).

[12] P. Collins, Dynamical Systems 19, 1 (2004).

[13] P. Collins, Experimental Mathematics 14, 75 (2005).

[14] P. Collins, Dynamical Systems 20, 369 (2005).

[15] C. Jung and H. Scholz, J. Phys. A 20, 3607 (1987).

[16] B. Ruckerl and C. Jung, J. Phys. A 27, 55 (1994).

[17] B. Ruckerl and C. Jung, J. Phys. A 27, 6741 (1994).

[18] C. Lipp and C. Jung, J. Phys. A 28, 6887 (1995).
[19] C. Lipp and C. Jung, Chaos 9, 706 (1999).

[20] A. Emmanouilidou, C. Jung, and L. E. Reichl, Phys. Rev. E 68, 46207 (2003).

[21] A. Emmanouilidou and C. Jung, Phys. Rev. E 73, 16219 (2006).

[22] J. Bergamin, T. Bountis, and C. Jung, J. Phys. A 33, 8059 (2000).

[23] K. A. Mitchell, J. P. Handley, B. Tighe, J. B. Delos, and S. Knudson, Chaos 13, 880 (2003).

[24] K. A. Mitchell, J. P. Handley, J. B. Delos, and S. Knudson, Chaos 13, 892 (2003).

[25] K. A. Mitchell, J. P. Handley, B. Tighe, A. Flower, and J. B. Delos, Phys. Rev. Lett. 92, 73001 (2004).

[26] K. A. Mitchell, J. P. Handley, B. Tighe, A. Flower, and J. B. Delos, Phys. Rev. A 70, 43407 (2004).

[27] K. A. Mitchell and J. B. Delos, Physica D 221, 170 (2006).

[28] K. A. Mitchell and J. B. Delos, Physica D 229, 9 (2007).

[29] P. Hansen, K. A. Mitchell, and J. B. Delos, Phys. Rev. E 73, 66226 (2006).

[30] J. B. Delos and K. A. Mitchell, Few-Body Syst. 38, 181 (2006).

[31] K. A. Mitchell and D. A. Steck, Phys. Rev. A 76, 31403 (2007).

[32] K. A. Mitchell, Physica D 238, 737 (2009).

[33] S. Wiggins, Introduction to Applied Nonlinear Dynamical Systems and Chaos (Springer, New York, 2003).

[34] W. Fulton, Algebraic Topology: A First Course (Springer, New York, 1995).

[35] J. A. Novick, Ph.D. thesis, College of William and Mary, 2009.

[36] See Supplemental Material at http://link.aps.org/supplemental/ 10.1103/PhysRevE.85.016206 for a derivation of the dynamical equations acting on the 16-basis. 